\title{
Discrete Angle Radiative Transfer 3. Numerical Results and Meteorological Applications
}

\author{
ANTHONY DAVIS ${ }^{1}$, PHILIP GABRIEL ${ }^{2}$, SHAUN LOVEJOY, DANIEL SCHERTZER ${ }^{1}$ AND \\ GEOFFREY L. AUSTIN
}

Physics Department, McGill University, Montréal, Québec, Canada

\begin{abstract}
In the first two installments of this series, various cloud models were studied with angularly discretized versions of radiative transfer. This simplification allows the effects of cloud inhomogeneity to be studied in some detail. The families of scattering media investigated were those whose members are related to each other by scale changing operations that involve only ratios of their sizes ("scaling" geometries). In part 1 it was argued that, in the case of conservative scattering, the reflection and transmission coefficients of these families should vary algebraically with cloud size in the asymptotically thick regime, thus allowing us to define scaling exponents and corresponding "universality" classes. In part 2 this was further justified (by using analytical renormalization methods) for homogeneous clouds in one, two, and three spatial dimensions (i.e., slabs, squares, or triangles and cubes, respectively) as well as for a simple deterministic fractal cloud. Here the same systems are studied numerically. The results confirm (1) that renormalization is qualitatively correct (while quantitatively poor), and (2) more importantly, they support the conjecture that the universality classes of discrete and continuous angle radiative transfer are generally identical. Additional numerical results are obtained for a simple class of scale invariant (fractal) clouds that arises when modeling the concentration of cloud liquid water into ever smaller regions by advection in turbulent cascades. These so-called random " $\beta$ models" are (also) characterized by a single fractal dimension. Both open and cyclical horizontal boundary conditions are considered. These and previous results are contrasted with plane-parallel predictions, and measures of systematic error are defined as "packing factors" which are found to diverge algebraically with average optical thickness and are significant even when the scaling behavior is very limited in range. Several meteorological consequences, especially concerning the "albedo paradox" and global climate models, are discussed, and future directions of investigation are outlined. Throughout this series it is shown that spatial variability of the optical density field (i.e., cloud geometry) determines the exponent of optical thickness (hence universality class), whereas changes in phase function can only affect the multiplicative prefactors. It is therefore argued that much more emphasis should be placed on modeling spatial inhomogeneity and investigating its radiative signature, even if this implies crude treatment of the angular aspect of the radiative transfer problem.
\end{abstract}

\section{INTRODUCTION}

\subsection{Radiative Transfer in Inhomogeneous Media}

A basic problem in meteorology, climatology, and remote sensing is to relate the highly variable properties of such geophysical fields as cloud or rain with the properties of their radiation fields at various wavelengths. If a distribution of scatterers is specified, and the sources of illumination given, the radiation field generated after multiple scattering can in principle be determined; the inverse is not possible in general. Most attempts to relate these fields have therefore proceeded by specifying some distribution of cloud scatterers (i.e. liquid water content with some droplet size distribution) and then computing the radiation fields, typically using Mie theory locally and Monte Carlo techniques globally.

For some time the term "inhomogeneous" atmosphere in the radiative transfer literature was synonymous with a vertically stratified system of atmospheric layers which can be described within the context of plane-parallel geometry; see Lenoble [1977] for an extensive review. There is no doubt that

\footnotetext{
1 Now at Établissement d'Études et de Recherches Météorologiques, Centre de Recherches en Météorologie Dynamique, Météorologie Nationale, Paris, France.

2 Now at CIRA, Dept. of Atmospheric Science, Colorado State University, Fort Collins.
}

Copyright 1990 by the American Geophysical Union.

Paper number 89JD03148.

0148-0227/90/89JD-03148\$05.00 stratification is present in the atmosphere, but ignoring horizontal variability is a very extreme assumption: if applicable, the variability of satellite imagery would only be due to spatial distribution of surface albedo. This simple fact has created the need to better understand "mutidimensional" radiative transfer, a term to which we prefer "horizontally inhomogeneous" for reasons that will soon become clear. Incidentally, inhomogeneous ground reflectance is sufficient to create horizontal gradients everywhere in the radiation field; Malkevich [1960], Diner and Martonchik [1984a,b], Tanré et al. [1981, 1987], and others approach this important problem in various ways.

Returning to clouds, the prime source of variability of atmospheric radiation, there as been a sustained interest in treating even simple geometrical shapes (e.g., cubes, cylinders, spheres) with various methods while maintaining internal homogeneity; see for example, Busygin et al. [1973], McKee and Cox [1974], Davies [1976, 1978], Barkstrom and Arduini [1977], Cogley [1981], Welch and Zdunkowski [1981a], Preisendorfer and Stephens [1984], and Stephens and Preisendorfer [1984]. Developments by Avaste and Vaynikko [1974], Busygin et al. [1977], Aida [1977], Glazov and Titov [1979], Titov [1979, 1980], Davies [1984], and others consisted in arranging these homogeneous clouds into periodic or uniformly random arrays in order to simulate cloud fields. Striated systems (one-dimensional horizontal variability) have always attracted some attention, e.g., Giovanelli [1959], Weinman and Swartztrauber [1968], van Blerkom [1971], Romanova [1975], Wendling [1977], Romanova and Tarabukhina [1981], and Stephens [1986, 1988a]. Inasmuch as they can be distinguished from the random array problem mentioned above, relatively little attention has been paid to systems with internal inhomogeneities; authors such as Mosher 
[1979] and Welch [1983] have adopted a deterministic approach whereas Welch et al. [1980], Gabriel et al. [1986], Stephens [1988b] and Cahalan [1989] have treated media involving random optical density fields. This is to be contrasted with the idea of ensemble-averaging of the (nonlinear) radiative responses associated with simple homogeneous cloud geometries in an attempt to model the effect of spatial variability [Mulloama et al., 1975; Ronnholm et al., 1980; Welch and Zdunkowski, $1981 b, c]$. See also Davis et al 1990, Lovejoy et al 1990 for calculations of scaling exponents characterizing the ensemble properties of multifractal clouds.

It should be mentioned that many of the cloud models discussed above were optically quite thin: $(1-g) \tau$, which is a measure of nonlinearity in the radiative response, was in the range 1 to $10 ; \mathrm{g}$ and $\tau$ designate as usual the asymmetry factor and optical thickness respectively. They almost always had quite modest inhomogeneities, limited to a very narrow range of scales. Not surprisingly, only small (but systematic) changes in the albedo were found in comparison with completely homogeneous plane-parallel clouds. It will be shown further on that arbitrarily large effects are achieved by thick enough clouds that merely lose light through their sides; it will also be seen that quite thin $(\tau=50,1-g \approx 0.85)$ clouds yield large differences with respect to plane-parallel predictions if enough internal inhomogeneity is present even when the range of scales is limited to a factor of 32 .

This succinct review is only concerned with the problem of multiple scattering with (usually collimated) external illumination. For completeness, we should mention the work of several geophysicists and many astrophysicists concerned with the effects of spatial variability (in more than one direction) of internal (thermal) sources for continuum or coherent transfer [Harshvardan et al., 1981; Stephens and Preisendorfer, 1984; Stephens, 1986] as well as of the frequency redistribution function for (incoherent) spectral line transfer [Jones and Skumanich, 1980].

\subsection{Need for Fractal Cloud Models}

There are many theoretical reasons, as well as considerable empirical evidence, supporting the idea that over wide ranges in scale, the statistical properties of clouds are invariant under a scale changing operation. Scale invariant (scaling) systems are associated with power law behaviour and complex fractal structures since over the corresponding range, the system has no characteristic size. Theoretically, we expect atmospheric fields including clouds to be scaling since the governing dynamical equations have no characteristic length between the outer (planetary) scale and the inner (viscous) scale of order one millimeter. Furthermore, the radiative transfer equation contains no intrinsic scale either. Below, we shall consider observationally based motivations.

Empirical (aircraft) energy spectra of cloud liquid water content (such as the power law energy spectra obtained by King $\mathrm{et}$ al. [1981]) are scaling (or scale invariant) in form, and broadly support the idea that at least over wide ranges in scale that clouds are fractal [Lovejoy, 1982; Rhys and Waldvogel, 1986; Kuo et al., 1988; Welch et al., 1988a,b; Yano and Takeuchi, 1990; Cahalan and Joseph, 1989]. For reviews, see Lovejoy and Schertzer [1986], Schertzer and Lovejoy [1988, 1990] and Cahalan [1989]. There have been some reports of scale breaking, but these may well be due to the use of monofractal rather than multifractal analysis techniques combined with use of a very small number of samples; see Lovejoy and Schertzer [1990a] for a discussion of this difference as well as a critical reevaluation of previous analyses. In any case, systematic studies of scaling and its limits in the atmosphere still have not been undertaken and the basic issues are still open.

At the very least, cloud scaling is complex. In this regard,
Gabriel et al. [1988] analyzed several infrared and visible radiation fields captured by GOES, over ranges from 8 to 512 $\mathrm{km}$; they found that the intense and weak regions have different scaling exponents (i.e. clouds are "multifractal"). Schertzer and Lovejoy $[1987 a, b]$ show theoretically that under fairly general circumstances the entire multifractal spectrum or (co)dimension function, can itself be characterized by three parameters which define multifractal "universality classes". Unlike fractal dimensions which provide purely geometric characterizations of sets, these parameters characterize the dynamical generator of the process. Lovejoy and Schertzer [1990b] refine the analysis of Gabriel et al. [1988] and estimate the three parameters for infrared and visible cloud/surface intensities. A further complexity in the scaling is empirically discussed in Lovejoy et al. [1987] (in radar rain fields) showing that the appropriate scale changing operator is not simply a zoom (self-similarity), but involves stratification as well (and a new "elliptical dimension"); Schertzer and Lovejoy [1983, 1985a] argue that it also involves differential rotation due to the Coriolis force and associated with cloud "texture". Multiple scaling and anisotropy are thus likely to be fundamental ingredients of realistic cloud models.

\subsection{Overview}

In the following section, most of the simple homogeneous and fractal clouds discussed in part 2 [Gabriel et al., this issue] are studied numerically in both discrete angle (DA) and continuous angle radiative transfer following the guide-lines prescribed in part 1 [Lovejoy et al., this issue]. More precisely, we exploit systematically the scaling ansatz in the asymptotic regime (we obtain quantitative estimates of various scaling exponents) as well as the exact DA similarity relations (only isotropic scattering is needed) and, most importantly, we give preference to Monte Carlo over diffusion methods. We then define a "packing factor" $(\xi)$ as the ratio of optical thicknesses of fractal and/or (horizontally) finite clouds to that of their plane-parallel counterparts with identical albedoes; the basic results can be summarized by $\xi \approx \tau^{\delta}$ where $\delta>0$ is the "packing exponent". In section 4 , we numerically examine random monofractal " $\beta$ model" clouds, showing that they too have reflected fields with considerably lower (average) albedoes than the corresponding plane-parallel layers.

We then outline the implications of our findings for the interpretation of satellite images, radiation budget and climate models as well as the "cloud albedo paradox", among several other possible fields of application. We then discuss possible improvements such as the use of more realistic fractal models, including the effect of statification (without leaving a scaling framework). Finally, we argue that most research into atmospheric radiative transfer has attributed too much importance to the subtleties of the angular distribution of intensity patterns due to ever more realistic phase functions exiting unrealistically homogeneous cloud layers. While DA systems may be crude as far as their treatment of interaction between intensities in different directions, their spatial variability can be much more realistic and interesting than in the usual plane-parallel systems. In any case, the universality conjecture (shown to hold here in most systems), means that for many thick cloud properties, the limitations of DA transfer due to its simplified coupling of intensities in direction space, will be secondary.

\section{MONTE CARLO STUDIES OF RADIATIVE TRANSFER IN SCALING CLOUDS}

\subsection{Review and Statement of Objectives}

Throughout this series of papers our objective as been to investigate the radiative behavior of certain simple examples of "scaling" clouds, i.e., those in which features at small and large 
scales are related by a scale changing operation that only depends on scale ratios, not absolute sizes. In these systems, the albedo $(R)$ and transmittance $(T)$ are nondimensional scaling functions of the optical thickness as the latter tends to infinity. This arises because the only characteristic (optical) length scale based on the scattering/absorbing process itself (the "diffusion length" $\approx\left[\left(1-\omega_{0}\right)\left(1-\omega_{0} g\right)\right]^{-1 / 2}$, where $\omega_{0}$ is the single-scattering albedo) diverges when scattering becomes conservative $\left(\boldsymbol{\omega}_{0} \rightarrow 1\right)$; hence the radiative properties of the system depend only on the dimensionless optical thickness. When the latter becomes much greater than one, the only critical value for a naturally defined nondimensional parameter, we expect the following algebraic (rather than exponential) behaviour:

$$
\begin{aligned}
& T-T^{*} \approx h_{T}(\mathrm{P}) \tau^{-v_{T}} \\
& R^{*}-R \approx h_{R}(\mathrm{P}) \tau^{-v_{R}}
\end{aligned}
$$

where $\mathrm{P}_{i k}$ is the DA phase function for scattering from directions $i$ into $k$; as customary, the matrix $P$ is only considered to be a function of of the scalar product $i \cdot k$. This constraint allowed us to enumerate exhaustively the acceptable DA systems which can be unambiguously designated with the following notation: $\mathrm{DA}(d, n)$, where $d=1,2,3$ is the dimension of the embedding space and $\mathrm{n}$ the number of beams. As indicated in $(1 a)-(1 b)$ and in accord with the exact DA(d, 2d) similarity relations obtained in part 1 for orthogonal systems, $h_{T}$ and $h_{R}$ are $P$ dependent prefactors whereas $v_{T}$ and $v_{R}$ are $P$ independent (i.e., universal) scaling exponents; signs are chosen to make all these parameters positive. Alternatively, $(1 a)$ and $(1 b)$ can be viewed as two-term asymptotic expansions of the radiative response functions as $\tau \rightarrow \infty$.

Since the DA phase functions are special cases of the general continuous angle phase functions, it is natural to conjecture that if universal continuous angle exponents exist, they should equal the DA exponents. This conjecture is all the more plausible given that the universal result for plane-parallel geometry $v_{R}=v_{T}=1$ (independent of phase function) is already well established in the radiative transfer literature; in the non-plane-parallel cases studied numerically here, it is also seems to be generally true. It should be clear, however, that the boundary conditions are important in determining the universality class. In $\operatorname{DA}(d, 2 d)$ systems and fixed boundary conditions, we showed in part 1 that (for $d>1$ ) the universality classes can be defined by the sign of $p q$ where $q$ and $p$ are simply related to the first and second Legendre coefficients of the DA phase function (the zeroth coefficient, $\boldsymbol{\omega}_{0}$, being in this conservative case unitary). For physically interesting phase functions, we have $2>q>p>0$.

In part 2, we approached the scaling relations $(1 a)$ and $(1 b)$ from the point of view of renormalization. According to this picture, these arise because near the thick cloud fixed point $\left[R^{*}, T^{*}\right]=[R(\infty), T(\infty)]$, the nonlinear map $[R(\tau), T(\tau)] \rightarrow[R(2 \tau), T(2 \tau)]$ can be approximated linearly by a matrix whose coefficients are independent of the DA phase function. Because the largest eigenvalue of this matrix is strictly less than unity, repeated multiplication of the matrix (corresponding to approaching the fixed point in a small neighbourhood) reduces the magnitude of errors as well as differences due to various initial phase function choices; the exponents $v_{R_{-}}$and $v_{T}$. are therefore universal (in this case, in the precise sense of nonlinear systems theory). The "-" signs are used to remind us that renormalization converges onto the universality class corresponding to $p q<0$ and unphysical (negatively valued) phase functions. Before reaching the thick cloud regime by iteration of the map, appreciable absolute differences in transmission or reflection coefficients can build up because of the instability of the thin cloud fixed point which initially amplifies such differences; the prefactors $h_{R-1}, h_{T \text { - }}$ will therefore depend on the phase functions. Indeed they will depend primarily on $(1-g)$ according to the approximate renormalization approach applied to the linear (thin cloud) regime; the exact $\mathrm{DA}(d, 2 d)$ similarity relations of part 1 dictate a dependence on $q / p$.

\subsection{Horizontally Finite Homogeneous Clouds in Two and Three Dimensions}

Because we were only interested in total fluxes through external cloud boundaries, and not in the internal radiation fields, standard Monte Carlo methods are sufficiently accurate and efficient. Although alternative methods, such as numerically solving the spatially discretized DA equations (cf. appendix) although have the advantage of giving the DA intensity fields everywhere, they involve large grids when the thick cloud limit is required, since the elementary cell optical thickness $\left(\tau_{0}\right)$ must be «1 everywhere (see section 3.2 and Appendix A of part 1). Approaches based on the $d$-dimensional diffusion equation should be avoided, as it is likely to be a poor approximation to the radiative transfer equation in general, i.e. when gradients in optical density are everywhere important; worse, it will generally have different universality classes, as shown in part 1 in analogy with the problem of phase transitions in percolating conductor/superconductor mixtures.

We therefore performed simulations in two dimensions on a square cloud with sides varying from $1 / 8$ up to 512 (in optical units). Two extreme examples of isotropic conservative scattering were used: continuous angle (with normal injection) and the orthogonal DA equivalent (i.e., the DA $(2,4)$ system with $t=r=s=1 / 4$ ). In both cases, the standard (exponential) photon free path distribution was employed; this means that, unlike the calculations presented in part 2 , no cell structure is needed. In the DA case, we have therefore numerically solved

$$
\left[\mathbf{A}_{y} \frac{\partial}{\partial y}+\mathbf{A}_{z} \frac{\partial}{\partial z}\right] I=-(1-\mathbf{P}) I
$$

where distances are measured in optical units (i.e., we take $\kappa \rho=1$ ), the (formal) vector $I(y, z)=\left(I_{+y}, I_{-y}, I_{+z}, I_{-z}\right) T$ contains the four beam intensities (the superscript " $T$ " designating matrix transposition), and

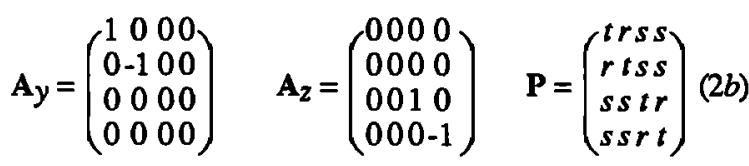

cf. part 1, section 3.3. The boundary conditions are $I_{+y}(0, z)=I_{-y}(\tau, z)=I_{-z}(y, \tau)=0$ and $I_{+z}(y, \tau)=1$ for $0 \leq y, z \leq \tau$. In the continuous angle case, the corresponding problem is described by

$$
(s \cdot \nabla) I_{s}(x)=-\left\{I_{s}(x)-\int_{0}^{2 \pi} p\left(\theta_{s s^{\prime}}\right) I_{s}(x) d \theta_{s s^{\prime}}\right\}
$$

where $\cos \theta_{s s^{\prime}}=s^{\prime} s^{\prime}$ and $I_{\underline{\underline{s}}}(\underline{\mathrm{x}})$ is the two-dimensional intensity at $\boldsymbol{x}=(y, z)$ in direction $s$; for conservative and isotropic scattering, we simply put $p\left(\theta_{s s^{\prime}}\right)=1 / 2 \pi$. The boundary conditions are $I_{s}(0, z)=I_{s}(\tau, z)=I_{s}(y, \tau)=0$ and $I_{s}(y, \tau)=\delta(s+n)$ for $0 \leq y, z \leq \tau$ and $s \cdot n<0$, where $n$ is the outgoing normal of the cloud's boundary. Notice the one-to-one correspondance of terms in (2a) and (3). Albedo $(F=R)$ and transmittance $(F=T)$ are defined by 


$$
F=\int_{0}^{\tau} d y \int_{s \cdot n>0} s \cdot n I_{s}(y, z) d \theta_{s s^{\prime}}
$$

for $z=0, \tau$ respectively; of course, no angular integration is needed in the DA formulation. Results are presented in Figure 1a: as predicted in parts 1 and 2, both transmittance curves are well described by $v_{T}=1$ and all the reflectance data are compatible with $v_{R}=3 / 4$ for large values of $\tau$. This supports the existence of broad universality classes; for other continuous angle phase functions with forward peaks in $d=2$, see Davis et al. [1989].

Figure $1 b$ shows three-dimensional Monte Carlo results from Davies [1976] and Gabriel [1988], using an isotropic and a more realistic (Diermenjian $\mathrm{Cl}$ drop size distribution at $0.45 \mu \mathrm{m}$

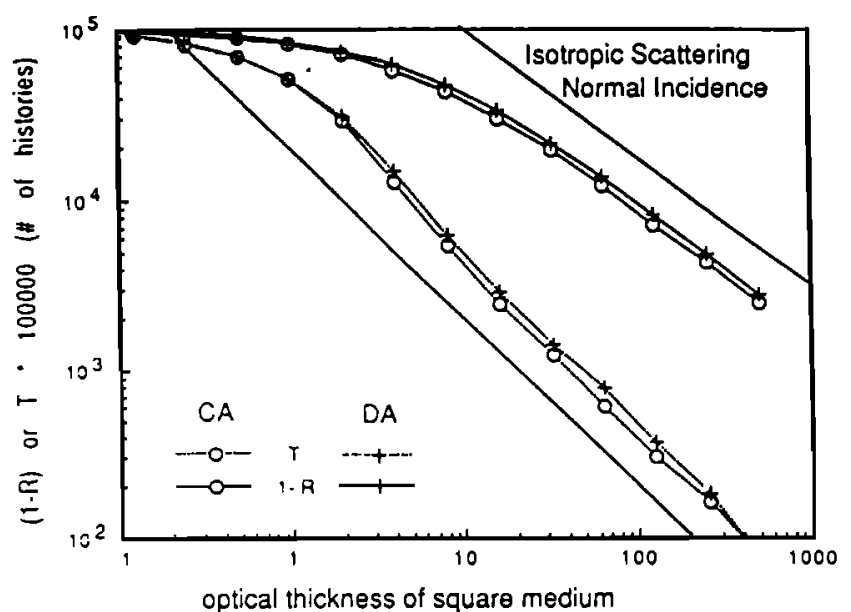

Fig. $1 a . T \times 10^{5}$ or $(1-R) \times 10^{5}$ versus $\tau$ for isotropic $(g=0)$ scattering in continuous angle and DA $(2,4)$, obtained by Monte Carlo simulation on normally illuminated squares. The reference lines show the asymptotic slopes $v_{R}=3 / 4$ and $v_{T}=1$. Here and in Figures $3 a-$ $3 c$, the intrinsic uncertainties of the method can be estimated as the square root of the ordinate.

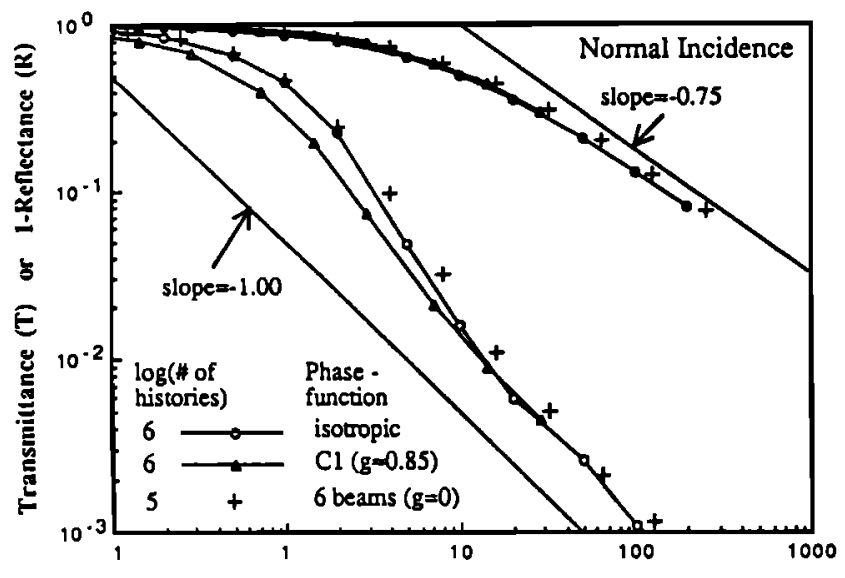

rescaled optical thickness of cube, (1-g)"tau

Fig. $1 b . T$ or $(1-R)$ versus $(1-g) \tau$ for anisotropic (Deirmenjian C1 drop size distribution, $g \approx 0.85$ ) and isotropic scattering in continuous angle or DA $(3,6)$, obtained by Monte Carlo simulation on normally illuminated cubes with $10^{6}$ photons (except for DA, where $10^{5}$ histories were used). yielding $g \approx 0.85$ ) phase function as well as new results using an isotropic six-beam DA phase function, i.e. the DA $(3,6)$ system with boundary conditions and definitions (4) above appropriately extended. It is notable that $1-R$ and $T$ obey approximately universal functions of $(1-g) \tau$ over the whole range from thin to thick regimes; this is also the case in $d=2$ as mentioned by Davis et al. [1989]. The same conclusion about universality as in $d=2$ follows, and the absolute asymptotic slopes are again found to be $v_{T}=1$ and $v_{R}=3 / 4$. This last result shows that the exponents associated with physical $p q>0$ and unphysical $p q<0$ are different in different d-dimensional spaces, since in Part II we obtained $v_{R_{-}}=1 / 2$ for $\operatorname{DA}(3,6)$ and $v_{R_{-}}=3 / 4$ for $\operatorname{DA}(2,4)$.

\subsection{Fractal Clouds with Dimension $D=\log 3 / \log 2=158 \cdots$}

The deterministic fractal clouds studied in this subsection are the same natural extensions of the internally homogeneous cloud models that were introduced in part 2: rather than being homogeneous over a set with dimension equal to that in which the cloud is embedded they are "fractally homogeneous", i.e., homogeneous over a (fractal) set with dimension less than that of the embedding space. By construction, these clouds contain horizontal inhomogeneities (that have an equal vertical extension) on all scales, from the size of the unit cell to that of the complete cloud, whereas the internally homogeneous horizontally finite clouds studied above present inhomogeneity at a very specific scale (i.e., the size of the cloud). Of course, such models are still too homogeneous to be realistic; multifractal measures (requiring an infinite number of dimensions for their specification) not monodimensional sets will undoubtedly be eventually necessary. It should be noted that following the convention in statistical physics, we have built the fractal clouds from an inner scale upwards to larger scales, whereas in turbulence (and multifractals in general), it is the small scale limit which is of primary interest and a different limiting procedure is appropriate (see Davis et al 1990, Lovejoy et al 1990 for more details).

Figure 2 illustrates the first seven steps in the construction of the specific model used in the following; notice that the number of cells increases as $3^{n}=\left(2^{n}\right)^{D}=(\text { physical size })^{D}$ where $D=\log 3 / \log 2=1.58 \cdots$ is the fractal dimension of the cloud. In our Monte Carlo experiment, the only need for spatial discretization was to specify the distribution of scattering versus nonscattering elements, which are characterized by their optical thicknesses $\tau_{0}(>0)$ and 0 , respectively. Single (active) cell optical thickness $\tau_{\mathrm{o}}$ was chosen as $1 / 8,1 / 2$, or 2 ; up to 12 steps into the construction procedure illustrated in Figure 2 were used (i.e. the cloud is on a grid of up to $4096 \times 4096$ points). The (space-averaged) optical thickness after $n$ steps is $\tau_{0}(3 / 2)^{n}$, as can be seen directly or by setting $\lambda=2$ and $C=d-D$ in (16) below. For simplicity, scattering is taken as isotropic either $\mathrm{DA}(2,4)$ or continuous in direction space; finally, $10^{5}$ photons were injected normally from above; see Figure 2. The equations obeyed by these systems are similar to those given in the previous subsection with left-hand sides multiplied by $\kappa \rho(x)$ equal to $\tau_{0}$ times the indicator function of the set depicted in Figure 2 (if distances would then be measured in units of cell size).

Figure $3 a$ shows the results of such calculations for the transmission law, the parameters in $(1 a)$ are found to be $T^{*}=0$, $v_{T} \approx 0.51$ (using the data for $\tau_{0}=2$ ). However Figures $3 b$ and $3 c$ corresponding to reflection show $R^{*} \approx 1 / 2$, its precise value must be estimated numerically along with $v_{R}$. The value $T^{*}=0$ can be understood quite easily since in thicker and thicker clouds, typical photons (that do not fall on a portion of cloud that is $\approx \tau_{0}$ thick) must traverse increasingly large optical distances in order to exit from the bottom of the cloud. 


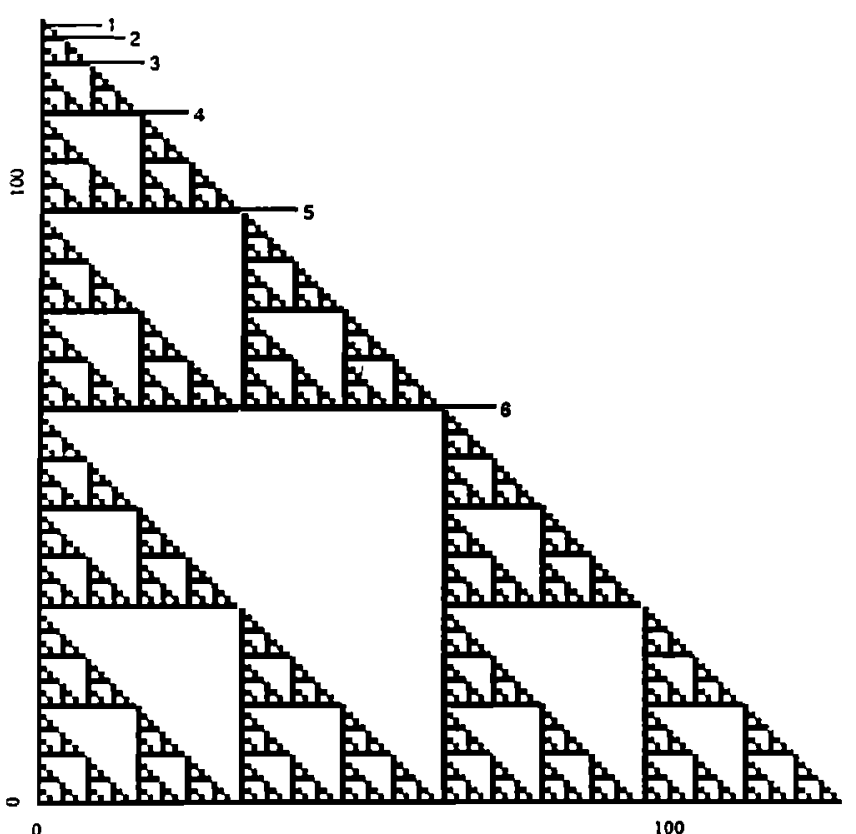

Fig. 2. Deterministic monofractal used as prototypical medium permeated by holes of all sizes, $N$ successive steps into the construction procedure can be visualized above the horizontal lines designated by $N$ (for $0 \leq N \leq 7$ ). For the purpose of all radiative transfer calculations presented here, illumination is from the top.

However, in the case of the albedo, most of the photons will be reflected after following only relatively short optical paths, even in very thick clouds. Furthermore, the cloud possesses a symmetry axis (at $45^{\circ}$, from the vertical from lower left to upper right comers in Figure 2), which combined with the isotropic phase functions used here, this ensures that roughly half of the (vertically incident) photons will escape from the top and half from the right-hand side of the cloud (if it is very thick). By the same token, we see that if the cloud were rotated $45^{\circ}$ counter clockwise in Figure 2, its $R^{*}$ would become 1; equivalently, rotate the angle of incidence $45^{\circ}$ clockwise and define $R$ as the flux contained in the semicircle within $\pm 90^{\circ}$ of the incident beam. Either way, we see that the boundary conditions (of illumination) have an obvious influence on the thick cloud limit. In order to determine the values of $R^{*}$ and $v_{R}$, it is convenient to graph the finite derivative of the albedo with respect to the natural log of (space-averaged) optical thickness against the albedo itself, thus avoiding a nonlinear three parameter fit. From $(1 b)$ one easily obtains

$$
\frac{\Delta R}{\Delta \ln \tau}=\frac{R(\tau)-R(2 \tau / 3)}{\ln 3 / 2}=\left[R^{*}-R(\tau)\right] \frac{(3 / 2)^{\mathrm{V}_{R}-1}}{\ln 3 / 2}
$$

Such a graph is shown in Figure 4; from the linear regression coefficients one obtains $v_{R}{ }^{*} \approx 0.46$, and $R^{*} \approx 0.53,0.54$ in the continuous and DA cases, respectively. As for the transmittance data, the exponents obtained are the same (to within numerical precision) for either phase function, supporting the hypothesis that universality extends from DA to continuous angle phase functions. The same (Monte Carlo) methods were used by Davis et al. [1989] to study the same cloud but with cyclical boundary conditions (where $T=1-R$ ). They report a minor violation of universality of the scaling exponents, finding $v_{T}^{*} \approx 0.41$ for continuous angle and $v_{T}^{*} \approx 0.50$ for $\mathrm{DA}(2,4)$ systems both normal and inclined in Figure 2 . The violation is

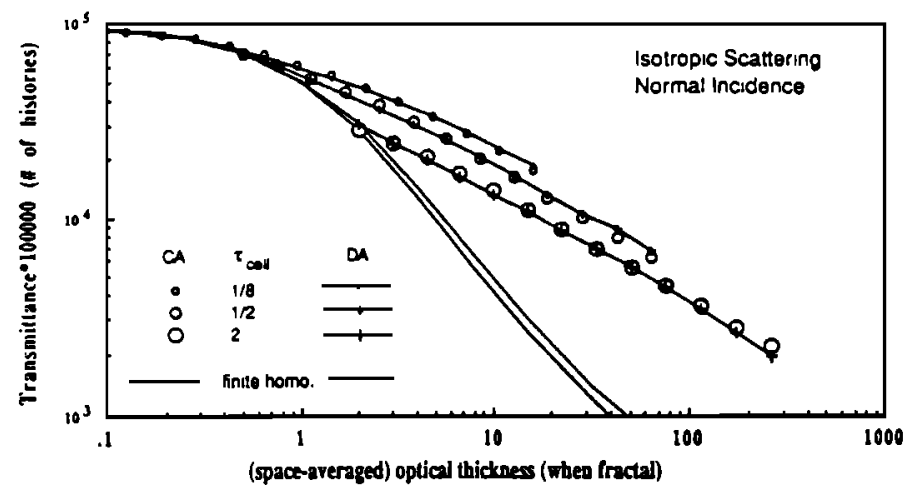

Fig. 3a. Transmittance versus spatially averaged optical thickness for the $D=1.58 \cdots, d=2$ cloud using continuous angle and $\mathrm{DA}(2,4)$ Monte Carlo. The data points correspond to 0 through 12 construction steps, respectively $1 \times 1$ to $4096 \times 4096$ grid points; three different cell optical thicknesses were used $(1 / 8,1 / 2,2)$. The absolute slope for the most opaque cells is $v_{T} \approx 0.51$. Corresponding transmittance curves are also shown for homogeneous squares (which yield $v_{T}=1.00 \cdots$ and somewhat different prefactors for either phase function).

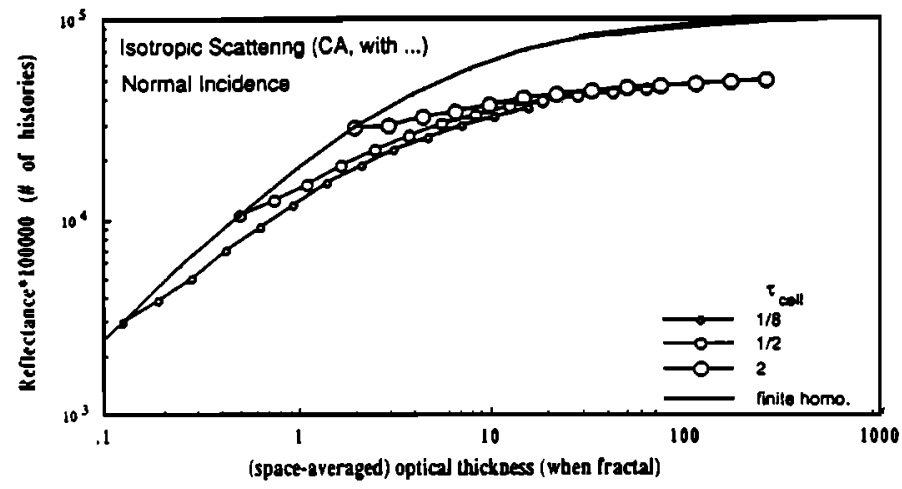

Fig. 3b. Same as Figure $3 a$ but for reflectance (through the "top") in continuous angle radiative transfer. Notice that $R^{*}=1$ for the homogeneous squares and $R^{*}<1$ for their inhomogeneous counterparts.

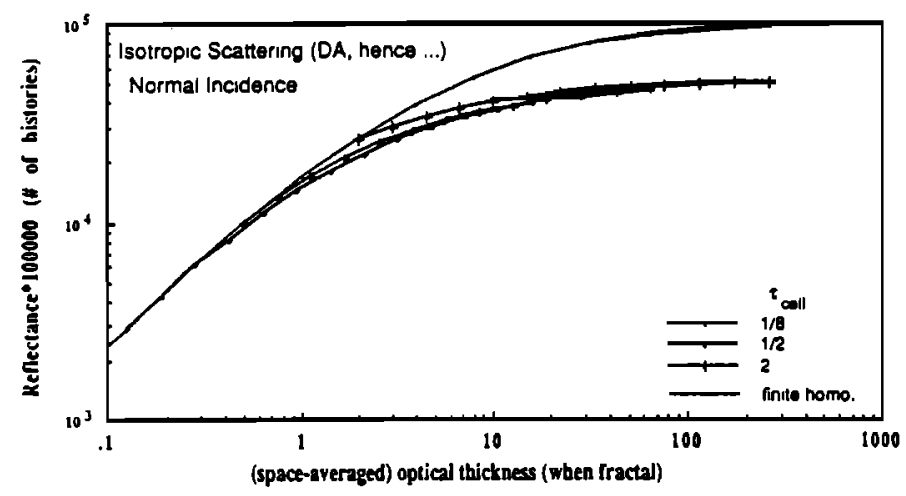

Fig. $3 c$. Same as Figure $3 b$ but for the $\mathrm{DA}(2,4)$ system.

minor because the $\mathrm{DA}(2,6)$ system is in the same universality class as continuous angle radiative transfer.

It is noteworthy that all the qualitatively correct predictions of the renormalization approach are retrieved in somewhat different guise: we have here $v_{R}$ (cyclic) $=v_{T}$ (cyclic) $<1$ and the effect of opening the boundary conditions is again to decrease $T\left(v_{T}\right.$ goes from 0.41 to 0.51 for $\tau_{0}=2$ ) and, to a greater extent, $R$ 


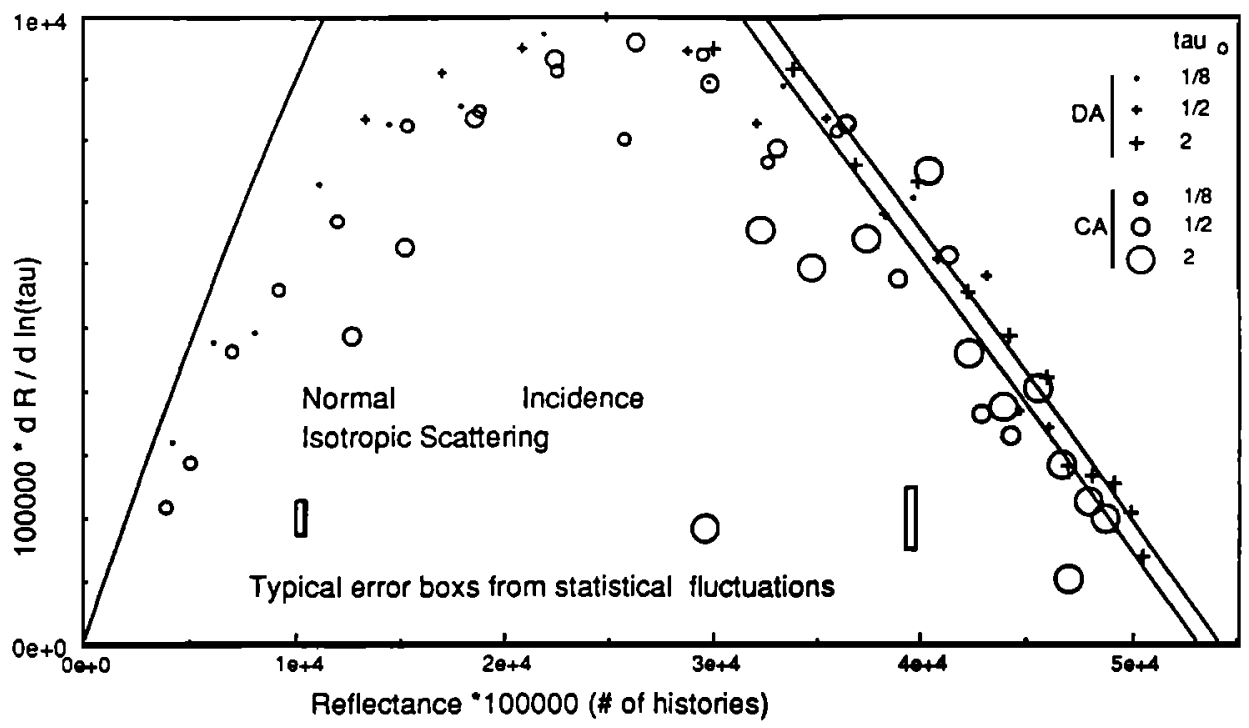

Fig. 4. Plotting technique described in text for the objective determination of $R^{*}$ and $v_{R}$. Straight lines at right are linear regressions for continuous angle and DA results, yielding $v_{R} \approx 0.46$ in both cases, whereas $R^{*} \approx 0.53$ for continuous angle and $R^{*} \approx 0.54$ for $\operatorname{DA}(2,4)$. The slightly curved line at left corresponds to plane-parallel media in the two-flux approximation or (equivalently) the DA(1,2) model.

although this happens via $R^{*}$ (which goes from 1 to $\approx 1 / 2$ ) rather than $v_{R}$ (which actually increases from 0.41 to 0.50 ) whereas renormalization (part 2) simply predicts $v_{R}$ (open) $<v_{R}$ (cyclic) $=v_{T}$ (cyclic) $<v_{T}$ (open $)<1$. The failure of renormalization to predict a change in $R^{*}$ is traceable to the assumption that losses are identical on either side of a highly asymmetrical fractal.

\section{IMPLICATIONS FOR CLOUD - SOLAR RADIATION INTERACTION}

\subsection{Quantitative Measures of the Effect of Inhomogeneity: Packing Factors and Absorptivity Ratios}

The results presented above indicate that due to the nonlinearity of the intensity with respect to the optical properties, the albedo of the fractal cloud approaches its thick limit much more slowly than in a uniform cloud since then $v_{R}(1)=1$ and $v_{R}(2)=v_{R}(3)=3 / 4$ for $\operatorname{DA}(d, 2 d)$ with $d=1,2,3$ or continuous angle. In order to quantify this effect we define a "packing factor", denoted $\xi$, as the ratio of the (spatially averaged) optical thickness of fractal and plane-parallel (i.e., onedimensional) clouds with the same albedo. We can calculate it as follows. Recall that for the fractal,

$$
R^{*} 1.58-R_{1.58} \approx h_{1.58} \tau_{1.58}-\mathrm{v}_{R}(1.58)
$$

while for the plane-parallel reference cloud, we have:

$$
1-R_{1} \approx h_{1} \tau_{1}^{-1}
$$

Taking $R_{1}=R_{1.58}$ and defining $\xi$ as $\tau_{1.58} / \tau_{1}$, we obtain

$$
\xi \approx G \tau_{1.58}+H \tau_{1.58}{ }^{1-v_{R}(1.58)}
$$

where $G=\left(1-R^{*} 1.58\right) / h_{1}$ and $H=h_{1.58} / h_{1}$ will depend on the phase functions, unlike $v_{R}$, which is $>0$ and $<1$. When open boundary conditions prevail, we have $R^{*} 1.58<1$, the asymptotic behavior of $\xi$ will then be

$$
\xi \propto \tau_{1.58}
$$

However, for the fractal cloud with periodic boundary conditions (or illumination along the symmetry axis in Figure 2) as well as for the square and cubic geometries, we found $R^{*}{ }_{1.58}=R^{*}{ }_{2}=R^{*}{ }_{3}=1$. We therefore obtain in this case (dropping subscripts):

$$
\xi \propto \tau^{1-v_{R}}
$$

In general, we may write

$$
\xi \propto \tau^{\delta}
$$

where $\tau$ is the large optical thickness of the (fractal) cloud and $\delta>0$ is the packing exponent; clearly, $\xi$ diverges with $\tau$, since $\delta>0$. The same is true for any horizontally finite cloud (such as a square or cubic cloud) in which $v_{R}(d)<1$ for $d>1$ due to losses through the sides. We can also anticipate that $\xi \rightarrow 1^{+}$for $\tau \rightarrow 0^{+}$; to see this return to the definition of $\xi$ and notice that (say) in figure $1 \mathrm{~b}$ (for the cubic cloud) we have $1-R_{1}=T_{1}<1-R_{3}$ (implying that $R_{1}=1-T_{1}>R_{3}$ ) for all values of $\tau$.

An equivalent way of comparing the horizontally finite or fractal clouds to their plane-parallel counterparts is to define the "absorptivity" as $1-R$ and calculate the ratio of these (apparent) absorptivities $\left(1-R_{1.58}\right) /\left(1-R_{1}\right)$ for clouds with the same optical thicknesses. Again using the fractal cloud as an example, we obtain

$$
\frac{1-R_{1.58}}{1-R_{1}} \approx G \tau+H \tau^{1-v_{R}(1.58)}
$$

the only difference with eq. (3.3) being that here we have $\tau=\tau_{1.58}=\tau_{1}$ (rather than $R_{1.58}=R_{1}$ ); absorbtivity also diverges at least as fast as $\tau^{1-v_{R}(1.58)}$ when $\tau \rightarrow \infty$.

We obtain similar results if the packing factors and absorption ratio are redefined by replacing the reference plane-parallel cloud by a reference square (even cubic) cloud, i.e., internally homogeneous but with losses through the sides: strictly positive packing exponents. Hence divergence of packing factors and absorptivities would still follow as a consequence of the presence of holes in horizontally extended clouds. This results from the 
fact that $v_{R}(1)=1>v_{R}(2) \approx v_{R}(3)>v_{R}(1.58)$ and that $R^{*}(1)=R^{*}(2)=R^{*}(3)=1 \geq R^{*}(1.58)$.

Furthermore, the presence of internal inhomogeneities is sufficient (and apparently necessary) to cause divergence of the corresponding ratios based on transmitted fluxes. This, in turn, comes from the fact that $v_{T}(1)=v_{T}(2)=v_{T}(3)=1>v_{T}(1.58)$ and that $T^{*}(1)=T^{*}(2)=T^{*}(3)=T^{*}(1.58)=0$. In the fractal cloud, this substantial increase in transmission could be thought of as a manifestation of the "channeling" of photons through regions of lesser optical density as described by Cannon [1970]. The same remark applies to internally homogeneous horizontally finite media where the photons are channeled toward the sides (which can be viewed as vertical interfaces with an optical vacuum).

\subsection{The Importance of Boundary Conditions}

In part 2 and section 2 above, we showed that leakage of light from the sides in two or three dimensions was sufficient to yield albedo exponents less than the corresponding plane-parallel values, yielding $\delta>0$. As expected, the effect of internal inhomogeneities discussed in connection with the 1.58...-dimensional cloud leads to a much stronger divergence when compared with plane-parallel media; even using the homogeneous square media as benchmark yields $\delta>0$. Another way of isolating the effect of inhomogeneity alone is to consider systems which are periodic in the horizontal direction (cyclic boundary conditions).

From the results of part 2, we can see that the effect of imposing cyclic boundary conditions on the fractal medium has been to increase the albedo exponent and to decrease the transmission exponent. The Monte Carlo results from subsection 2.2 above show that albedo is changed in the same direction but via $R^{*}$ (from $\approx 1 / 2$ to 1 ) even though the corresponding exponent actually changes in the opposite direction (from 0.46 to 0.41 ). The transmittance is also boosted, this time, via a decrease in its scaling exponent (from 0.51 to 0.41 ). Since these values are all substantially below the plane-parallel values $\left(R^{*}=1\right.$, $v_{R}=v_{T}=1$ ), we again conclude that inhomogeneity is sufficient in itself to yield diverging packing factors by enhancing transmittance at the expense of reflectance.

This is also the case in the homogeneous two- or three-dimensional (square or cubic) clouds, where imposing periodic boundary conditions would lead to plane-parallel geometry and results in identical reflection and transmission exponents equal to 1 . This again calls for a substantial increase of $v_{R}$ (from $3 / 4$ to 1 ) whereas transmittance is only increased through $h_{T}$. We notice that renormalization methods remain qualitatively correct, predicting an increase in $v_{R}$ -

From the point of view of the total radiative response $F$, the parameters that influence the thick cloud values are primarily $F^{*}$ and then $h_{F}$ and $v_{F}$. We see that albedo $(F=R)$ always reacts "more" to any form of horizontal inhomogeneity (including finiteness) than transmittance $(F=T)$. This can be understood by recalling that, due to highly asymmetric boundary (illumination) conditions, levels of (total) radiant energy are much higher near the top of a thick cloud than near its bottom because the contribution of low orders of scattering have the same up/down asymmetry.

\subsection{More on Packing Factors}

It was shown in the previous subsections that optical media with various geometries exhibit systematic differences in their radiative responses to external illumination when compared with plane-parallel clouds with equivalent optical thicknesses. The packing factor introduced to quantify this effect could be formally written

$$
\xi=\frac{\tau}{F_{\text {ref }}^{-1}[F(\tau)]}
$$

where $F$ is one of the response functions ( $T$ or $R$ ) of optical thickness $\tau$ (eventually space averaged) and $F_{\text {ref }}$ is the same optical response for some reference medium. In other words, $\tau_{\text {eff }}=F_{\text {ref }}[F]$ is the effective optical thickness needed to obtain a response $F$ from the reference medium and $\xi$ is the ratio $\tau / \tau_{\text {eff. }}$. Notice that $\xi$ is an unambiguous measure of the effect of geometry perturbation, since $F$ and $F_{\text {ref }}$ are both monotonic. Furthermore, plane-parallel geometry provides a natural point of reference, since it is so widely used.

We have seen that, for the various types of horizontal inhomogeniety considered, using plane-parallel theory as the benchmark yields $\xi(\tau)>1$ for $\tau>0$. A consequence of this regularity of $\xi$ is that the homogencous/plane-parallel distribution of optical density is not average but extremal in the sense that it is undoubtably the most efficient way of reducing the flux of radiant energy as measured by (total) transmittance or, equivalently, exciting a diffusely reflected radiation field. This means that, except in very artificial (laboratory) situations, using plane-parallel theory to fit data gives a lower bound rather than an (unbiased) estimate of $\tau$.

It is not hard to see that the nonlinearity of $F$ with respect to $\tau$ can be exploited to yield a nontrivial (>1) statistical analog of the "packing" of cloud mass (by inhomogeneity effects) if only the ensemble-averaged quantities are known. In analogy with definition (13), we write

$$
\xi_{s}=\frac{\langle\tau}{F^{-1}[<F(\tau)>]}
$$

where angle brackets denotes ensemble averaging and again $F=R, T$ or any other monotonic radiometric measure of $\tau$ at given optical parameters (say, $\bar{\sigma}_{0}$ and $g$ ), geometry and illumination conditions. The main difference between the definitions of $\xi$ and $\xi_{s}$ is that the former calls for two different functions of the same argument, whereas the latter uses the same one before and after ensemble averaging. This was carried out for simple plane-parallel geometry with truncated Gaussian [Mullaama et al., 1975] or lognormal [Ronnholm et al., 1980] variability models for $\tau$, whereas Welch and Zdunkowski [1981b,c] use an exponential distribution of cylinders. Part of the motivation behind these studies is to simulate the effect of stochastic cloud fields, i.e., spatial variability, on observable cloud properties. This procedure is only justified in the case of extremely tenuous fields of horizontally finite clouds, in which case radiative interaction between clouds can be neglected. Above all, $\xi_{\mathrm{s}}$ is not to be confused with $\langle\xi\rangle$, obtained from (13), which is used to characterize the properties of the cloud models discussed in the following section and for which inhomogeneity is intrinsically stochastic.

We have argued that there is no clear-cut difference between an extended inhomogeneous cloud and a cloud field. Realistic cloud models must therefore incorporate not only an element of stochasticity but a continuously tuneable parameterization for spatial correlations. The simplest possible random cloud model is (the spatial equivalent of) "white" (uncorrelated) noise [Welch et al., 1980], but by definition, it lacks the long-range clustering observed in real clouds. Multifractals (including monofractals as a special case) are naturally associated with " $1 / f$ " noise and correlation lengths equal to the outer scale of the variability field. A randomized version of our fractal model which, by construction, incorporates this feature is presented in the next section and applied to DA radiative transfer. 


\section{DA RADIATIVE TRANSFER IN RANDOM FRACTAL ClOUDS}

\subsection{Turbulent Cascades of Passive Scalar Clouds}

Up until now, we have studied cloud models that were geometrical rather than dynamical. So far most efforts at dynamic cloud/radiation modeling have been at (thermal) infrared wavelengths using statistical closure techniques [see Simonin et al.,1981; Schertzer and Simonin, 1983; Coantic, 1978; and the astrophysical literature, starting with Spiegel, 1957]. The closure approach is limited primarily by its inability to properly handle intermittency (i.e. extreme variability). In the cloud radiation problem, this will be a serious shortcoming, since we have seen that even simple fractal intermittency leads to diverging packing factors. When applied to thick scattering atmospheres, the closure approach is further limited by the fact that unlike its thermal emission counterpart, the multiple-scattering source function is not directly expressible in terms of a local temperature since the corresponding radiation field is extremely far from thermodynamic equilibrium [Essex, 1984]. Another approach is to use standard numerical modeling techniques for directly simulating all the dynamical fields and their interactions (including radiative) [e.g., Welch, 1983]. The difficulty here is that even the largest scale dynamical models (which have a factor of the order of only 100 in their range of scales) do not contain a sufficient range of scales to allow intermittency to build up significantly. Indeed, the basic process responsible for the concentration of energy, and other conserved fluxes into smaller and smaller regions of space, is the turbulent cascade, and the existing models do not have enough degrees of freedom to allow such cascades to develop. For instance, they do not display the expected $k^{-5 / 3}$ energy spectra for wind and passive scalar fields ( $k$ designates wave number). Existing models are dominated by energy injection at large scales and dissipation at small scales, with insignificant intermediate "inertial" or cascade ranges. On the one hand, the empirical cloud densities are highly intermittent [Tsay and Jayoweera, 1984] and scale roughly as $k^{-5 / 3}$ over several orders of magnitude [King et al., 1981]. On the other hand, we have shown that (in our deterministic models) it is precisely this scaling intermittency which leads to large radiative effects (large packing factors). We will therefore use stochastic cascade models which are scaling by construction and are specifically designed to produce strong intermittency.

In hydrology, stochastic models of rain have been developed for some time (see, e.g., Waymire and Gupta [1981] for an early review, and the special issue of the Journal of Geophysical Research, volume 92(D8), for a recent survey). While some of these could be used for cloud modeling, we concentrate here on the subclass of fractal models. The simplest of these [Lovejoy and Mandelbrot, 1985] involves the addition of a large number of "pulses", but has the drawback that it lacks physical motivation and is characterized by a single fractal dimension. A much more physically based (multi)fractal model is the passive scalar turbulent model outlined by Schertzer and Lovejoy [1987a,b]. This model has the same symmetries (e.g. scaling) as the dynamical (partial differential) equations goveming passive scalar advection in incompressible turbulence, as well as the same cascade phenomenology.

The simplest special case (developed for modeling the nonlinear energy flux density $\varepsilon=\partial v^{2} / \partial t$, where $v$ is the wind velocity vector) is called the " $\beta$ model" [Mandelbrot , 1974; Frisch et al., 1978] and, as pointed out by Schertzer and Lovejoy [1983], is the only case involving a single fractal dimension. As illustrated in Figure $5 a$ and applied to passive scalar variance flux density ( $\chi=\partial \rho^{2} \partial t$ where $\rho$ is the passive scalar density), the $\beta$ model creates a highly variable (intermittent) field by randomly concentrating the large scale flux density (schematically indicated in the figure by the large uniform square) into subregions modeling the nonlinear breakup of an eddy into subeddies. The quantities $\chi, \varepsilon$ are fundamental, since they are exactly conserved by the nonlinear terms in the equations governing passive scalar advection, and are thus expected to be scale invariant down to viscous scales (typically of the order of millimeters).

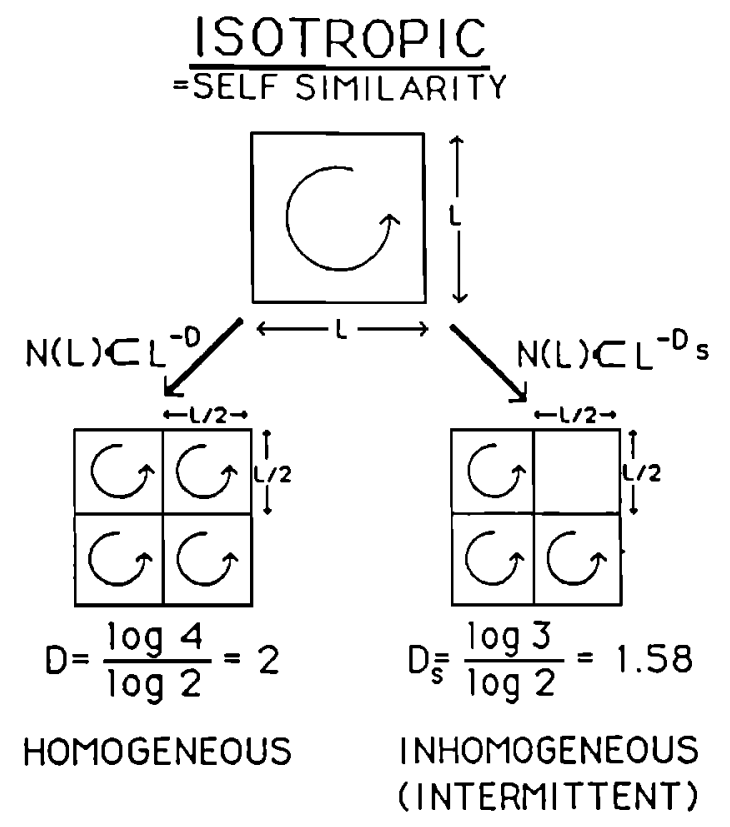

Fig. 5a. A schematic representation of how turbulent cascades treat the breakup of a single eddy (represented by the central square) via nonlinear interactions during a single step of the cascade process. Both schemes shown here are isotropic; the left-hand side is homogeneous, and the right hand side is an intermittent $\beta$ model.

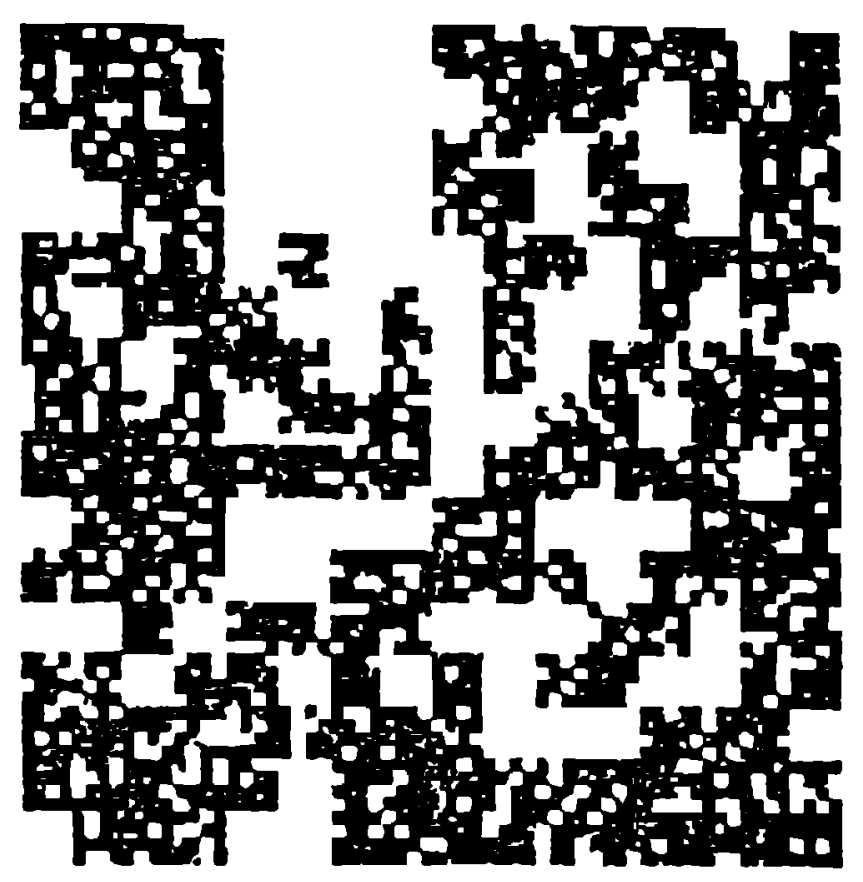

Fig. 5b. An example of a canonical $\beta$ model in $d=2$ with a $4 \times 4$ breakup per cascade step, showing the first four steps; black indicates the remaining "alive" regions. Fractal dimension is set at $D=1.73$, i.e., at each step, each cell has a probability $4^{-0.2} 7=0.688 \cdots$ of remaining alive where $0.27=C=2-D$. 


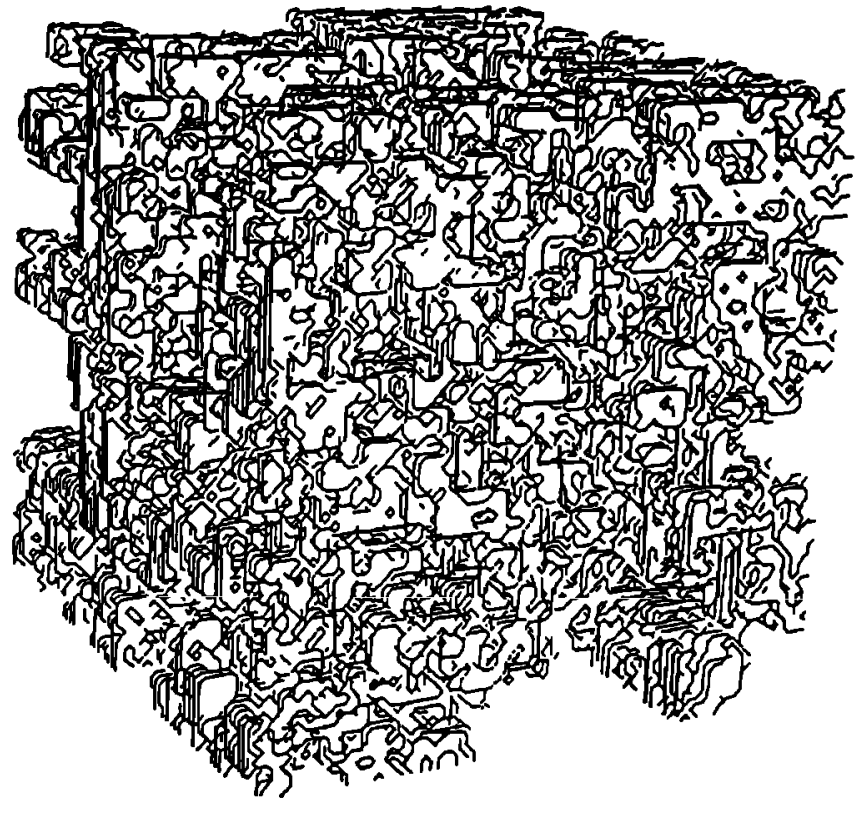

Fig. 5c. A perspective view of a three-dimensional $\beta$ model cloud with $D=2.6(C=0.4)$ on a $32^{3}$ grid (i.e., five cascade steps with a dividing ratio of 2 ).

Once the statistically stationary conserved quantities $\chi, \varepsilon$ are given, the fluctuations in density are described by the CorrsinObukhov scaling law:

$$
\Delta p \approx \varepsilon^{-1 / 6} \chi^{1 / 2} \Delta x^{1 / 3}
$$

where $\Delta p$ is the (mean) difference in density between two points separated by a distance $\Delta x$. Various methods of numerically implementing this phenomenology are possible [Schertzer and Lovejoy, 1987b; Wilson et al., 1990]. Here, for simplicity, we model the optical density $(p \kappa)$ directly as a cascade quantity, ignoring the additional scaling implied by the $\Delta x^{1 / 3}$ factor in (15). A binomial process randomly determines if a subeddy is "alive" with probability $\lambda^{-C}$ or "dead" with probability $1-\lambda-C$. $\lambda>1$ is the scale ratio between successive steps of the cascade process, and $C \geq 0$ is the codimension with $C=d-D$, where $d$ is the dimension of space in which the cascade occurs and $D$ is the fractal dimension of the live regions; for instance, $\lambda=2$, $d=2$ and $D=\log 3 / \log 2$ in figure $5 a$. If the eddy is alive, the flux density is increased by a constant factor $\lambda C$, such that the ensemble-averaged total (area integrated) flux is conserved. In the limit of a large number of cascade steps, the flux is almost surely every where zero except on a fractal set, with dimension $D$. The example in Figure $5 b$ corresponds to $\lambda=4, d=2$ and $D=1.73$ (after three steps into the cascade), whereas Figure $5 c$ shows a perspective illustration of the $\beta$ model embedded in a three-dimensional space such as those used below for the purpose of DA radiative transfer calculation.

A slightly more general scheme, is the " $\alpha$ model" proposed by Schertzer and Lovejoy [1983]. The same binomial distribution is used but instead of being multiplied by $\lambda C$ or 0 , the multiplicative increments are taken to be $\lambda C / \alpha>1$ or some other strictly positive constant $\lambda-C / \alpha^{\prime}$ chosen so that $\angle \rho \cap O=1$ (the $\beta$ model is retrieved formally with $\alpha \rightarrow 1, \alpha^{\prime} \rightarrow 0$ ). In analogy with statistical mechanics, such models are said to be "canonical". An extremely restrictive variant is also possible: the "microcanonical" cascade in which energy (flux) is exactly conserved at each step. This is unphysical, since in the small scale limit, it implies flux conservation at each point; see Schertzer and Lovejoy [1990] for more details about these limitations. Cahalan's [1989] one-dimensional model uses a microcanonical cascade with $\lambda=2, \lambda-\mathrm{C}=1 / 2$ and $\lambda \mathrm{C} / \alpha=1.3$ (hence $\lambda-\mathrm{C} / \alpha^{\prime}=0.7$ ). In Davis et al 1990 , Lovejoy et al 1990, we obtain exact results for direct transmittance for a family of microcanonical $\alpha$ models of which the 1.58 model above, and Cahalan's 1989 model are special cases.

\subsection{DA(3, 6) Radiative Transfer in $\beta$ Model Fractal Clouds}

The $\beta$ model described above was used to obtain cloud geometry, and the DA $(3,6)$ system on a cubic lattice was used to determine the resulting radiation field by an over-relaxation method of iteration (see the appendix). We used $\lambda=2$ and $n=5$ cascade steps, thus generating a $32^{3}$ grid which was roughly the maximum possible with our computer resources (six intensities must be specified at each grid point). The single-cell transfer coefficients $(T, R, S)$ were obtained through standard (continuous angle) Monte Carlo methods and phase functions corresponding to a Deirmendjian $\mathrm{C} 1$ drop size distribution (at $0.45 \mu \mathrm{m}$ wavelength, $g \approx 0.85$ ) for a cube of optical thickness $\tau_{0}$ calculated in such way as to maintain the ensemble/spaceaveraged optical thickness constant throughout the experiment at $\langle\tau\rangle=50$. This mean optical thickness is given by

$$
<>=\tau_{0}\left(\lambda^{n}\right)^{1-C}
$$

For each value of the codimension $C(=3-D), 10$ realizations of the systems were used, each of which has a specific (spaceaveraged) optical thickness yielding a packing factor $\xi$ by solving the plane-parallel reflection law for optical thickness, given the (space-averaged) albedo of the model; in short, (16) is ensembl-averaged. In Figure 6, the mean (and standard deviation) of $\xi$ is plotted (logarithmically) against $C$, which varies from 0.1 to 0.8 . This corresponds to an ensemble- and space-averaged cloud optical thickness to cell optical thickness ratio $\left(<\tau>/ \tau_{0}\right)$ varying from 22.625 to 2.00 . In turn, this means that $(1-g) \tau_{0}$ varies from 0.33 to 3.75 , i.e., mainly in the linear $(T \approx 1)$ regime where spatially discretized and continuous DA calculations compare very well quantitatively. The packing factor

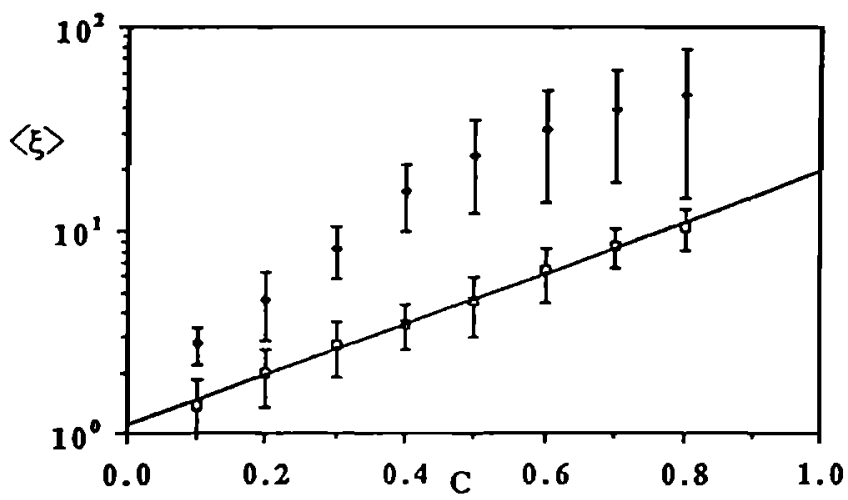

Fig. 6. Numerical estimates of ensemble-averaged packing factors $(\langle\xi\rangle)$ estimated for a cubic cloud having cyclic and open horizontal boundary conditions (bottom and top curves, respectively) as a function of codimension $(C)$ using $\mathrm{DA}(3,6)$ radiative transfer. Spaceand ensemble-averaged optical thickness is maintained at 50; cell optical thickness is thus $50 / 32^{1-C}$. See (16), which determines cell transfer coefficients (via standard Monte Carlo simulation for Dermeinjian's C1 drop size distribution at $0.45 \mu \mathrm{m}$ under normal incidence). 
is seen to increase with the sparseness of the medium as measured by $C$. When the cyclical horizontal boundary conditions are removed, $\langle\xi\rangle$ increases considerably as expected, since the albedo is reduced due to side "leakage".

Using an analysis technique called "functional box-counting" [Lovejoy et al., 1987], Gabriel et al. [1986] give numerical evidence that the radiation fields associated with $\beta$ model clouds are multifractal in spite of the monodimensional nature of the cloud field. This means that the fractal dimensions for the increasingly intense regions of the albedo field are not constant, but decrease. This is in accord with satellite data analyzed by Gabriel et al. [1988] and Lovejoy and Schertzer [1990b].

\section{APPLICATION TO SEI ECTED ATMOSPHERIC RADIATION PROBLEMS}

We have argued that radiative transfer in clouds depends critically on the assumptions that are made about the variability, or lack of variability, of the optical medium. To date, most radiative transfer calculations are made with the totally ad hoc assumption that clouds are plane-parallel. The limitation to this special (and unrealistic) case considerably restricts meteorological and climatological applications of radiative transfer. Conversely, systematic studies of radiative transfer in systems with strong horizontal and vertical variability will help to overcome outstanding difficulties. Areas where our findings are likely to be particularly relevant are discussed briefly below. Other areas not singled out for discussion, but nevertheless of potential interest, include rainfall and cloud estimation algorithms, radiative transfer theory, stochastic cloud modelling and nuclear winter studies.

\subsection{The Cloud Albedo Paradox}

We have seen that virtually any scaling family of clouds will be expected to have different thick cloud scaling exponents than the plane-parallel family, with the latter expected to yield the absolute maximum value. In terms of the packing factor $\xi$, this immediately leads to the general result $\delta>0$, where $\delta$ is the packing exponent introduced in section 3 , hence

$$
\xi(\tau) \approx \tau^{\delta} \rightarrow \infty \text { as } \tau \rightarrow \infty
$$

Although in reality, scaling will only hold over a limited range, cloud simulations presented in section 4 showed that $\xi$ can reach values of order 10 for cloud decks (cyclic boundary conditions) characterized by fractal dimension of 2.2 , using scaling regimes limited to a range of scales of only a factor 32 . Isolated clouds (open boundary conditions) attain the same value at dimension 2.7. This result has implications for the so-called "albedo paradox" [Wiscombe et al., 1984]. Stated simply, the paradox is that optical depths computed from seemingly reasonable liquid water profiles based on actual field measurements reach several hundred for only moderately thick clouds. However, in order to obtain consistency between the value of the planetary albedo $\approx 0.30$, and the globally averaged cloud cover (50\%), the clouds cannot have a mean albedo greater than $\approx 0.5$ [Paltridge and Platt, 1976], a value which for plane-parallel theory implies optical depths only of order 10 . Conversely, when plane-parallel models are used to approximate the radiative properties of real clouds (and they usually are), optical depths exceeding 100 imply visible albedoes greater than 0.9 , a value rarely if ever observed [Wiscombe et al., 1984]. Satellite observations also deduce smaller-than-expected optical depths [Twomey and Cocks, 1982]. The paradox vanishes once we leave the very artificial special case of plane-parallel media.

\subsection{The Interpretation of Satellite Images of Clouds}

Satellite imagery is widely used to make subjective judgements about meteorological features on a routine basis without any explicit account being taken of the sub-pixel variability. The dangers of such homogeneous, plane-parallel type interpretations of satellite data were underscored in Gabriel et al. [1988]. Defining a "feature" as a region of a satellite radiation field exceeding a radiance threshold, the hypothesis that such features are resolution-independent was tested. Except for extremely low thresholds, this hypothesis could be statistically rejected with very high levels of confidence (at both infrared and visible wavelengths over both mainly cloud free and cloud covered images using GOES data over Montreal). In as much as many algorithms for exploiting satellite data involve direct use of satellite radiances at various thresholds, these algorithms will contain hidden resolution dependencies. However, not all characteristics are resolution dependent; the "codimension function" determined by these authors is an example of such a statistic and should be used as the basis for a resolution independent approach towards quantitatively exploiting such data sets.

\subsection{Radiation Budget and Climate Models}

The need to relate the spatial and temporal variability of cloud and rain liquid water fields to the associated radiation fields at a variety of scales arises in both estimates of radiation budgets as well as in climate modeling; see Ramanathan et al. [1983, 1989]. The solution to these problems calls for an improved theory of cloud-radiation interaction and, among other things, an understanding of how radiance and albedo vary with spatial resolution. According to J.P. Blanchet and H. Barker (private communication,1989), preliminary general circulation model (GCM) runs using nontrivial packing exponents used to correct (plane-parallel) shortwave radiation packages yield better globally averaged planetary albedoes.

\section{FUTURE DIRECTIONS}

\subsection{Improvements in Cloud Modeling}

The chief reason for studying DA radiative transfer in the isotropic $\boldsymbol{\beta}$ model clouds described in the previous sections is simplicity. The calculations are (at least numerically) tractable, and the models involve a single parameter to describe inhomogeneity (i.e., fractal dimension). Below, we briefly sketch some obvious areas where realism must be added to the models. At least some of these improvements will be necessary before the models are sufficiently realistic to warrant detailed comparisons with measured cloud albedoes and transmission coefficients.

Stratification and texture. We have already noted that real clouds could not be exactly self-similar; otherwise extrapolation from a small roundish cloud to clouds hundreds of kilometers long, would lead to clouds hundreds of kilometers high. Lovejoy and Schertzer [1985], and Schertzer and Lovejoy [1985b, 1987a,b] outline a complete formalism called "generalized scale invariance" that allows differential rotation, stratification, as well as more general (nonlinear) transformations to be incorporated into a scale-invariant framework. They also introduce a new kind of "elliptical dimension" ( $\left.d_{\mathrm{el}}\right)$ that quantitatively describes the stratification. For example, it was argued that $d_{\mathrm{el}}=23 / 9=2.555 \cdots$ for the horizontal wind field. This means that rather than being completely isotropic $\left(d_{\mathrm{el}}=3\right)$ or completely flat $\left(d_{\mathrm{el}}=2\right)$, that the latter is "in between", i.e., characterized by a continuously scaling 
stratification. Using new data analysis techniques and radar data, Lovejoy et al. [1987] estimate $d_{\mathrm{el}}=2.22 \pm 0.07$ in the rain field, indicating that the latter is considerably more stratified than the former. It is therefore of some interest to analyze the effect of various degrees of stratification ( $\left.d_{\mathrm{el}}\right)$ on the radiation fields for both homogeneous and fractal clouds. From a modeling point of view, the difference between self-similar and stratified clouds is that the "zoom" relating small and large scales now involves compression (flattening) along the vertical. This is easy to achieve; for example, enlarge in the horizontal directions by $\lambda$ and in the vertical direction by only $\lambda H_{2}$ with $0 \leq H_{2} \leq 1$. In this case, the volume of a (homogeneous) cloud would increase by only $\lambda \lambda \lambda H_{z}=\lambda^{2+H} H_{z}=\lambda^{d} e l$, thus thicker and thicker clouds become flatter and flatter, with $d_{\mathrm{el}}$ somewhere between 2 and 3.

Continuous cascades and multifractal clouds. Other limitations of our cloud model are that it depends on scale changing with discrete factors and possesses only a single fractal dimension. A simple way of curing the latter problem would be to use an $\alpha$ model as discussed in section 4.1. A more physically realistic way which involves both continuous cascade processes and multiple fractal dimensions is to use the model described by Schertzer and Lovejoy [1987a,b]. Ultimately, we may expect that the "codimension function" of the optical density field (which specifies the entire spectrum of fractal sets needed to describe a field) should be related to that of the of the corresponding radiation field. Recall that the latter is a nonlinearly smoothed version of the former. The basic idea is that the codimension functions are the basic scale invariant objects; we expect the scale invariant connection between these two fields to be expressible in terms of these functions. Actually, the connection may even be simpler, since Schertzer and Lovejoy $[1987 a, b]$ have shown that the codimension functions belong to universality classes characterized by three parameters, i.e., they have simple "generators"; ultimately we seek relationships between these generators. The empirical determination of the functions at various wavelengths [Gabriel et al., 1988; Lovejoy and Schertzer, 1990], and the finding that they fall into universality classes predicted by continuous cascade processes, supports this idea.

\subsection{Improvements in Radiative Transfer Modeling}

Absorption and multiple wavelength properties. Most of the numerical and analytical methods used here and in parts 1 and 2 are valid in both conservative and non-conservative scattering. However, we have mainly studied the conservative scattering case because it yields both more interesting thick cloud behavior (power law rather than exponential decay) and because it is a good approximation to visible light scattering in real clouds. Although the effects of absorption at a single wavelength clearly require more study in their own right, such studies will also enable the overall wavelength dependence of radiative transfer from fractal clouds to be determined. Modeling the wavelength dependence is important in both (1) the standard "inversion" problem where radiances from various (remotely sensed) wavelengths are available and cloud characteristics such as mean optical thicknesses are sought and (2) estimating the "broad band" properties in spectral regions where absorption is important.

Multiple scattering in extremely variable random media (technical remarks). The multiple scattering source term in the radiative transfer equation effectively couples the radiation field's directional anisotropy and spatial inhomogeneity (hence gradients); see discussion in section 2.3 of part 1 . Up until now, the dominant method of overcoming this difficulty has been to restrict our attention to plane-parallel systems, allowing the intensity as a function of direction to be considered in great detail both analytically and computationally, exactly and approximately [Lenoble, 1977]. Given the unrealistic nature of the geometry, the most attractive of these plane-parallel models are of the approximate/analytical kind (especially when the computational overload must be kept to a minimum, e.g., in a GCM radiation module or a sensitivity study). Meador and Weaver [1980] propose a selection of two-stream models for fluxes (see Sobolev [1956] for intensity). At the same time the exact/computational models such as Stamnes et al. [1989] provide convenient benchmarks in validation studies [e.g. King and Harshvardan, 1986]. The only vehicle that allows spatial variability of arbitrary magnitude in all directions simultaneously is the computationally exact (but cumbersome) Monte Carlo method, exploited in section 2, and alternatively (and with more caution), finite differences, exploited in section 4 . This is very unsatisfactory mainly because these methods give no physical insight into the transfer processes, the fundamental equations are only used for interpretation of the results. Recent progress in the modeling of radiative transfer through arbitrary optical density fields while potentially maintaining the full angular complexity has been made by Stephens [1986, 1988a,b] using horizontal Fourier transforms. Because of the spatial anisotropy imposed by the specific (illumination) boundary conditions of the multiple scattering problem, vertical variability is best treated in real (possibly, Laplace) space. This results in beam and (Fourier) mode coupling in a formally plane-parallel problem and, in practice, this approach (coupled with invariant imbedding ideas) allows semi-analytical treatment of media with horizontal variability only.

We believe that the results obtained here in $\operatorname{DA}(d, n)$ systems show that the past emphasis on modeling angularly complex but spatially simple systems has been misplaced. Indeed, the powerful DA $(d, 2 d)$ similarity relations of part 1 (which seem to hold approximately in continuous angle systems) and the existence of universal phase function independent exponents points to the secondary role that the angular part of the problem appears to play with respect to the spatial part. The results obtained here therefore incite us to concentrate our attention on developing much more realistic models for the cloud fields.

However, it must be realized that the associated (even DA) radiative transfer problem will not be easily solved. While exploring the complications in the (two-stream) modeling of simply layered media analytically, Flateau and Stephens [1988] indicate the difficulty of modeling simultaneously horizontal and vertical inhomogeneity. Upon (vertical) integration, we are required to combine nonlinearly a large number of (large) random matrices which are infinitesmal or differ infinitesimally from unity. Any kind of vertical variability that cannot be absorbed into the definition of (average) optical depth, e.g., presence of layers where scattering is non-conservative (or, more interestingly, altitude dependent horizontal structure), means non-commutation of these matrices. In particular this implies that even a simple product cannot be done in general by exponentiating a sum of generators. In the case of multifractal cloud (optical density) fields, these matrices will furthermore be correlated over long distances like the optical density field itself. The Markov approach applied by Vanderhaegen [1986] to one-dimensional absorbing/emitting media may be of use for scattering media too, possibly along the lines suggested by Ronnholm et al. [1980]. At any rate, the DA simplification (used in conjunction with universality classification) will be welcome and the successes of scaling ideas in nonlinear dynamical systems theory, turbulence modeling and statistical physics encourage us to exploit them systematically in the context of radiative transfer. 


\section{Conclusions}

The major objective of this series of papers was to study radiative transfer in extremely inhomogeneous media. Scaling (fractal) cloud models were used both because these are more meteorologically realistic than their prevailing plane-parallel counterparts, and also because they form a simple class amenable to theoretical analysis. To make the problem tractable, we studied systems involving discrete angle phase functions, in which scattering only occurs in a finite number of directions. In these systems, the intensity field decouples into an infinite number of mutually independent families. Each family, which involves only interaction between a small number of angles, can then be studied separately. As usual, the equations can be solved on grids or by using Monte Carlo techniques. They can also be treated approximately using renomalization methods.

In this final part, we numerically investigated the scaling properties of the radiation fields associated with very simple homogeneous and fractal clouds, the latter generated by turbulent cascade models in which elementary cells end up either empty or full. To quantify the nonlinear effect of inhomogeneity, we defined a packing factor as the ratio of (space-averaged) optical thicknesses of fractal clouds to plane-parallel clouds. This packing of more liquid water into a cloud than is allowed by plane-parallel models is not exclusive to fractal clouds, leakage through sides is enough. Using $32^{3}$ grids, we showed that substantial deviations from plane-parallel behavior can occur even in systems involving scaling only over the limited range of a factor 32 in scale; we easily obtain packing factors of order 10, which is sufficient to explain the so-called "albedo paradox".

Although we discussed applications of these results to remote sensing and climate modeling, perhaps a more significant result will be a reappraisal of the relative importance given to the spatial and angular parts of the radiative transfer problem. Our results clearly indicate that if the spatial variability is not correctly modeled, arbitrarily large errors can occur. Conversely, even crude modeling of the angular aspect may be sufficient for many applications. Rather than developing sophisticated methods to account for the details of the angular part of radiative transfer in simplistic plane-parallel cloud models, we should be adding realism to the spatial distribution of cloud liquid water and concentrating on spatial and statistical aspects of the associated radiation field.

\section{APPENDIX: SPATIALLY DISCRETIZED EQUATIONS OF DA RADIATIVE TRANSFER AND THEIR NUMERICAL SOLUTION BY ITERATION}

In section 4 of this paper, much use is made of the equations of DA $(3,6)$ radiative transfer on a cubic lattice (see parts 1 and 2 for other possibilities):

$$
I_{i}(m)=\sum_{k} \sigma_{i k}(m) I_{k}(m-k)
$$

where $\sigma_{i k}(m)$ is the transfer matrix at lattice point $m$. We have here,

$$
\sigma=\left(\begin{array}{llllll}
T & R & S & S & S & S \\
R & T & S & S & S & S \\
S & S & T & R & S & S \\
S & S & R & T & S & S \\
S & S & S & S & T & R \\
S & S & S & S & R & T
\end{array}\right)
$$

in the (space-filling) $\mathrm{DA}(3,6)$ system where the $6 \boldsymbol{k}$ vectors are

$$
\left(\begin{array}{l}
1 \\
0 \\
0
\end{array}\right),\left(\begin{array}{c}
-1 \\
0 \\
0
\end{array}\right),\left(\begin{array}{l}
0 \\
1 \\
0
\end{array}\right),\left(\begin{array}{c}
0 \\
-1 \\
0
\end{array}\right),\left(\begin{array}{l}
0 \\
0 \\
1
\end{array}\right),\left(\begin{array}{c}
0 \\
0 \\
-1
\end{array}\right)
$$

$T$ is the transmittance of a (filled) cell, $R$ is its reflectance, and $S$ represents diffuse reflection through a side (not necessarily after single-scattering only) and $R+T+4 S=1$ for conservative scattering; $\sigma$ is, of course, the identity matrix at every empty cell in a fractal medium. The corresponding boundary conditions are unit "downward" intensity along horizontal boundary assigned to the top of the (grid describing the) cloud and zero "inward" intensity at all other boundaries, although intensity can be "recycled" from one vertical boundary back into its opposite, thus making the medium periodic.

Many methods of solution are possible, and we did not make an exhaustive study of the most efficient algorithms. The simplest method involves a simple iteration of (A1); it is known as Gauss-Seidel's and was that used by Mosher [1979] in the context of DA radiative transfer. Starting with an initial (guess) intensity field $I^{(0)}(m)$, we obtain the $(n+1)$ th iteration from the nth using

$$
I^{(n+1)} i_{i}(m)=\sum_{k} \sigma_{i k}(m) I^{(n)} k_{k}(m-k)
$$

The initial guesses used here were simply unit radiation (down) on the top and zero (in all directions) elsewhere. Iterating this equation is a numerically stable method of solution, since all the elements are positive, and the sum of any row (or column) of $\sigma$ is $\leq 1$ (alternatively, Appendix A of part 1 points out that the absolute eigenvalues of $\sigma$ are all $\leq 1$ ). Another related method that works well here is over-relaxed iteration; however, care must be taken in choosing the relaxation parameter.

Acknowledgments. We wish to thank R. Davies, H. Leighton, N. Sakellariou, A. Saucier, G. Stephens, T. Wam, A. Royer, N. O'Niell and our anonymous referees for many helpful discussions and comments.

\section{REFERENCES}

Aida, M., Scattering of solar radiation as a function of cloud dimensions ' and orientation, J. Quant. Spectrosc. Radiat. Trans., 17, 303-310, 1977.

Avaste, O.A., and G.M. Vaynikko, Solar radiation transfer in broken clouds, Izv. Acad. Sci. USSR Atmos. Oceanic Phys., 10, 1054-1061, 1974.

Barkstrom, B.R. and R.F. Arduini, The effect of finite size of clouds upon the visual albedo of the earth, in Radiation in the Atmosphere, edited by H.-J. Bolle, Science Press Princeton, 188-190, 1977.

Busygin, V.P., N.A. Yevstatov, and E.M. Feigelson, Optical properties of cumulus clouds and radiant fluxes for cumulus cloud cover, Izv. Acad. Sci. USSR Atmos. Oceanic Phys., 9, 1142-1151, 1973.

Busygin, V.P., N.A. Yevstatov, and E.M. Feigelson, Calculation of the direct, scattered and total solar radiant fluxes and their distribution in cumulus clouds, Izv. Acad. Sci. USSR Atmos. Oceanic Phys., 13, 184 190, 1977.

Cahalan, R.F., Overview of fractal clouds, paper presented at RSRM'87, Advances in Remote Sensing. pp 371-389, Edited by A. Deepak et al., A. Deepak, Hampton, Va., 1989.

Cahalan, R.F., Landsat observations of fractal cloud structure, in Scaling, Fractals and Non-Linear Variability in Geophysics, edited by D. Schertzer and S. Lovejoy, Kluwer, Hingham, Mass., in press, 1990.

Cahalan, R.F., and J.H. Joseph, Fractal statistics of cloud fields, Mon. Wea. Rev., 117, 261-272, 1989. 
Cannon, C.J., Line transfer in two dimensions, Astrophys. J., 161, 255264, 1970.

Coantic, M.F., Interaction between turbulence and radiation, AGARDograph, 232, 175-236, 1978.

Cogley, A.C., Initial results for multidimensional radiative transfer by the adding/doubling method, paper presented at the 4th Conference on Atmospheric Radiation, Am. Meteorol. Soc., Toronto, June 12-16, 1981.

Davies, R., The three dimensional transfer of solar radiation in clouds, Ph.D. thesis, 219 pp, Univ. of Wisc., Madison, 1976.

Davies, R., The effect of finite geometry on three dimensional transfer of solar irradiance in clouds, J. Atmos. Sci., 35, 1712-1724, 1978.

Davies, R., Reflected solar radiances from broken cloud scenes and the interpretation of scanner measurements, J. Geophys. Res., 89, 1259$1266,1984$.

Davis, A., P. Gabriel, S. Lovejoy, and D. Schertzer, Asymptotic laws for thick clouds, dimensional dependence - phase function independence, in IRS'88: Current Problems in Atmospheric Radiation, edited by J. Lenoble and J. F. Geleyn, pp. 103-106, A. Deepak, Hampton, Va., 1989.

Davis, A., Lovejoy, S., and D., Schertzer, Radiative transfer in multifractal clouds, in Scaling, Fractals and Non-Linear Variability in Geophysics, edited by D. Schertzer, and S. Lovejoy, Kluwer, Holland, in press, 1990.

Diner, D.J., and J.V. Martonchik, Atmospheric transfer of radiation above an inhomogeneous non-Lambertian ground, I, Theory, J. Quant. Spectrosc. Radiat. Transfer, 31, 97-125, $1984 a$.

Diner, D.J., and J.V. Martonchik, Atmospheric transfer of radiation above an inhomogeneous non-Lambertian ground, II, Computational considerations and results, J. Quant. Spectrosc. Radiat. Transfer, 32, 279-304, 1984b.

Essex, C., Radiation and the irreversible thermodynamics of climate, $J$. Almos. Sci., 41, 1986-1991, 1984.

Flateau, P.J., and G.L. Stephens, On the fundamental solution of the radiative transfer equation, J. Geophys. Res., 93, 11037-11050, 1988.

Frisch, U., P.L. Sulem, and M. Nelkin, A simple dynamical model of intermittent fully developed turbulence, $J$. Fluid Mech, 87, 719-724, 1978.

Gabriel, $P$. ., Discrete angle radiative transfer in uniform and extremely variable clouds, Ph.D. thesis, 160 pp. Phys. Dep., McGill Univ., Montréal, Québec, 1988.

Gabriel, P., S. Lovejoy, G.L. Austin, and D. Schertzer, Radiative transfer in extremely variable fractal clouds, paper presented at the 6th Conference on Atmospheric Radiation, Am. Meteorol. Soc., Williamsburg, Va., May 12-16, 1986.

Gabriel, P., S. Lovejoy, D. Schertzer, and G.L. Austin, Multifractal analysis of resolution dependence in satellite imagery, Geophys. Res. Lett., 15, 1373-1376, 1988.

Gabriel, P., S. Lovejoy, A. Davis, G.L. Austin, and D. Schertzer, Discrete angle radiative transfer, part 2 , Renomalization approach for homogeneous and fractal clouds, $J$. Geophys. Res., this issue.

Giovanelli, R.G., Radiative transfer in nonuniform media, Aust. J. Phys., 12. 164-170, 1959.

Glazov, G.N, and G.A. Titov, Average solar radiation fluxes and angular distribution in broken clouds, Izv. Acad. Sci. USSR Almos. Oceanic Phys., 15, 802-806, 1979.

Harshvardan, J.A. Wienman, and R. Davies, Transport of infrared radiation on cuboidal clouds, J. Atmos. Sci., 38, 2500-2513, 1981.

Jones, H.P., and A. Skumanich, The physical effects of radiative transfer in multidimensional media including models of the solar atmosphere Astrophys. J. Suppl., 42, 221-240, 1980.

King, W.D., C.T. Maher, G.A. Hepburn, Further performance tests on the CSIRO liquid water probe, J. Atmos. Sci., 38, 195-200, 1981.

King, W.D., and Harshvardan, Comparitive accuracy of selected multiple scattering approximations, J. Atmos. Sci., 43, 784-801, 1986.

Kuo, K.S., R.M. Welch, and S.K. Sengupta, Stuctural and textural characteristics of cirrus clouds observed using high spatial resolution Landsat imagery, J. Appl. Meteor., 27, 1242-1241, 1988.

Lenoble, J. (Ed.), Standard Procedures to Compute Radiative Transfer in a
Scattering Atmosphere, NCAR, Boulder, Colo., 1977. (Reprinted by A. Deepak, Hampton, Va., 1985.)

Lovejoy, S., The area-perimeter relation for rain and clouds, Science, 216 , 185-187, 1982.

Lovejoy, S., and B. Mandelbrot, Fractal properties of rain and a fractal model, Tellus, 37A, 209-232, 1985.

Lovejoy, S., and D. Schertzer, Generalized scale invariance in the atmosphere and fractal models of rain, Water Resour. Res., 21, 1233$1250,1985$.

Lovejoy, S., and D. Schertzer, Scale invariance, symmetries, fractals and stochastic simulation of atmospheric phenomena, Bull. Am. Meteorol. Soc., 67, 21-32, 1986.

Lovejoy, S., D. Schertzer, and A.A. Tsonis, Functional box-counting and multiple elliptical dimensions in rain, Science, 235, 1036-1038, 1987.

Lovejoy, S., and D. Schertzer, Multifractal analysis techniques and rain and cloud fields from $10^{-3}$ to $10^{6} \mathrm{~m}$, in Scaling, Fractals and Non-Linear Variability in Geophysics, edited by D. Schertzer and S. Lovejoy, Kluwer, Hingham, Mass., in press, 1990a.

Lovejoy, S., and D. Schertzer, Multifractals, universality classes and satellite and radar measurements of cloud and rain fields, $J$. Geophys. Res., 95, 2021-2034, 1990b.

Lovejoy, S., A. Davis, P. Gabriel, G.L. Austin, and D. Schertzer, Discrete angle radiative transfer, part 1 , Scaling and similarity, universality and diffusion, J. Geophys. Res., this issue.

Lovejoy, S., A. Davis, D. Schertzer, Radiative transfer in multifractal clouds: observations and theory, paper presented at the 7th Conference on Atmospheric Radiation, Am. Meteorol. Soc., San Francisco, July 2225, 1990.

Malkevich, M.S., An approximate method of allowing for horizontal albedo changes of the underlaying surface in problems of light scattering in the atmosphere, Bull. (Isv.) Acad. Sci. USSR, Geophys. Ser., no. 2, 1960.

Mandelbrot, B., Intermittant turbulence and self-similar cascades: Divergence of high moments and dimension of the carrier, J. Fluid Mech., 62, 331-350, 1974.

McKee, T.B., and J.T. Cox, Scattering of visible light by finite clouds, $J$. Atmos. Sci., 31, 1885-1892, 1974.

Meador, W.E. and W.R. Weaver, Two-stream approximations to radiative transfer in planetary atmospheres: A unified description of existing methods an a new improvement, J. Atmos. Sci., 37, 630-643, 1980.

Mosher, F.R., Visible flux across finite clouds, Ph.D. thesis, 109 pp, Univ. of Wisc., Madison, 1979.

Mullaama, R.Y., M.A. Sulev, V.K. Poldmaa, H.A. Ohvril, H.J. Niylsick, M.I. Allenov, L.G. Tchubakov, and A.F. Kuusk, Stochastic structure of clouds and radiation fields, NASA Tech. Transl., TT F-822, 1975.

Paltridge, G.W, and C.M.R. Platt, Radiative Processes in Meteorology and Climatology, 318 pp, Elsevier North-Holland, New York, N. Y., 1976.

Preisendorfer, R.W., and G.L. Stephens, Multimode radiative transfer in finite optical media, I. Fundamentals, J. Atmos. Sci., 41, 709-724, 1984.

Ramanathan, V., E.J. Pitcher, R. C. Malone, and M. L. Blackmon, The response of a spectral general circulation model to refinements in radiative processes, J. Atmos. Sci., 40, 605-630, 1983.

Ramanathan, V., E.J. Pitcher, R. C. Malone, and M. L. Blackmon, Cloud-radiative forcing and climate: Results from the Earth Radiation Budget Experiment, Science, 243, 57-63, 1989.

Rhys, F.S., and A. Waldvogel, Fractal shape of hail clouds, Phys. Rev. Lett., 56, L784-787, 1986.

Romanova, L.M., Radiative transfer in a horizontally inhomogeneous scattering medium, Izv. Acad. Sci. USSR Atmos. Oceanic Phys., 11. 509-513, 1975.

Romanova, L.M., and T.M. Tarabukhina, Light reflection by a sunlit horizontally inhomogeneous cloud, lzv. Acad. Sci. USSR Atmos. Oceanic Phys., 17, 19-26, 1981.

Ronnholm, K., M.B. Baker, and H. Harrison, Radiative transfer through media with uncertain or variable parameters, J. Atmos. Sci., 37, 1279 1290, 1980.

Schertzer, D., and S. Lovejoy, The dimension of atmospheric motions, 
paper presented at the IUTAM Symposium on Turbulence and Cheotic Phenomena in Fluids, 141-144, Kyoto Un., Japan, 1983.

Schertzer, D., and S. Lovejoy, The dimension and intermittency of atmospheric dynamics, in Turbulent Shear Flow, vol. 4, edited by B. Launder, Springer, New York, N.Y., pp 7-33, $1985 a$.

Schertzer, D., and S. Lovejoy, Generalized scale invariance in turbulent phenomena, P.C.H. Journal, 6, 623-635, 1985b.

Schertzer, D., and S. Lovejoy, Singularités anisotropes et divergence de moments en cascades multiplicatives, Ann. Math. Qué., 11, 139-181, $1987 a$.

Schertzer, D., and S. Lovejoy, Physically based rain and cloud modeling by anisotropic, multiplicative turbulent cascades, J. Geophys. Res., 92, 9693-9714, $1987 b$.

Schertzer, D., and S. Lovejoy, Multifractal simulations and analysis of clouds by multiplicative processes, Atmos. Res., 21, 337-361, 1988.

Schertzer, D., and S. Lovejoy, Scaling nonlinear variability in geodynamics: Multiple singularities, observables and universality classes, in Scaling, Fractals and Non-Linear Variability in Geophysics, edited by D. Schertzer and S. Lovejoy, Kluwer, Hingham, Mass., in press, 1990.

Schertzer, D., and O. Simonin, A theoretical study of radiative cooling in homogeneous and isotropic turbulence, in Turbulent Shear Flow, vol. 3. edited by L.J.S. Bradbury et al., Springer, New York, N.Y., pp. 262274, 1983.

Simonin, O., M.F. Coantic, and D. Schertzer, Effet du rayonnement infrarouge sur la turbulence de temperature dans l'atmosphère: Structure spectrale et taux de dissipation, C.R. Acad. Sci., 293, 245-248, 1981.

Sobolev, V.V., A Treatise on Radiative Transfer, 1956. (English translation, Van Nostrand Reinhold, New York, 1963.)

Spiegel, E.A., The smoothing of temperature fluctuations by radiative transfer, Astrophys. J., 126, 202-207, 1957.

Stamnes, K., S.C. Tsay, W.J. Wiscombe, and K. Jayaweera, A generalpurpose numerically stab!e computer code for discrete-ordinate-method radiative transfer in scattering and emitling layered media, in $I R S^{\prime} 88$ : Current Problems in Atmospheric Radiation, edited by J. Lenoble and J. F. Geleyn, pp. 448-453, A. Deepak, Hampton, Va., 1989.

Stephens, G.L., Radiative transfer in spatially heterogeneous, twodimensional anisotropically scattering media, J. Quant. Spectrosc. Radiat. Transfer, 36, 51-67, 1986.

Stephens, G.L., Radiative transfer through arbtrarily shaped media, I, General solution, J. Atmos. Sci., 45, 1818-1835, 1988a.

Stephens, G.L., Radiative transfer through arbtrarily shaped media, II, Group theory and closures. J. Atmos. Sci., 45, 1836-1838, 19886.

Stephens, G.L., and R.W. Preisendorfer, Multimode radiative transfer in finite optical media, II, Solutions. J. Almos. Sci., 41, 725-735, 1984.

Tanré, D., M. Herman, and P.Y. Deschamps, Influence of the background contribution upon space measurements of ground reflectance, Appl. Opt., 20, 3676-3684, 1981.

Tanré, D., P.Y. Deschamps, P. Duhaut, and M. Herman, Adjacency effect produced by the atmospheric scatering in thematic mapper data, $J$. Geophys. Res., 92, 12000-12006, 1987.

Titov, G.A., Solar radiation transfer modeling for cumulus cloud conditions, Izv. Acad. Sci. USSR Atmos. Oceanic Phys., 15, 432-436, 1979.

Titov, G.A., Statistical characteristics of short wavelength solar radiation in the presence of cumulus cloudiness, Izv. Acad. Sci. USSR Atmos. Oceanic Phys., 16, 500-505, 1980.
Tsay, S., and K. Jayaweera, Characteristics of arctic stratus clouds, $J$. Climate Appl. Meteor., 23, 584-596, 1984.

Twomey, S., and T. Cocks, Spectral reflectance of clouds in the near infrared: Comparisons of measurements and calculations, J. Meteorol. Soc. Jpn., 60, 583-592, 1982.

van Blerkom, D., Diffuse reflection from clouds with horizontal inhomogeneities, Astrophys. J., 166, 235-242, 1971.

Vanderhaegen, D., Radiative transfer in statistically heterogeneous mixtures, J. Quant. Spectrosc. Radiat. Transfer, 36, 557-561, 1986.

Waymire, E, and V.K. Gupta, The mathematical structure of rainfall representations, parts 1-3, Water Resour. Res., 17, 1261-1294, 1981.

Weinman, J.A., and P.N. Swartztrauber, Albedo of a striated medium of isotropically scattering particles, J. Atmos. Sci., 25, 497-501, 1968.

Welch, R.M., Radiation calculations based upon detailed microphysics, paper presented at the 5th Conference on Atmospheric Radiation, pp 505-506, Am. Meteorol. Soc., Baltimore, 1983.

Welch, R.M., and W.G. Zdunkowski, The effect of cloud shape on radiative characterisitics, Contrib. Atmos. Phys., 54, 482-491, 1981 a.

Welch, R.M., and W.G. Zdunkowski, The radiative characteristics of noninteracting cloud fields, I, Parameterization for finite clouds, Contrib. Atmos. Phys., 54, 258-272, $1981 b$.

Welch, R.M., and W.G. Zdunkowski, The radiative characteristics of noninteracting cloud fields, II, Calculations for cloud fields, Contrib. Atmos. Phys., 54, 273-285, 1981 c.

Welch, R.M., S. Cox, and J. Davis, Solar radiation and clouds, Meteorol. Monogr., 39, 1980.

Welch, R.M., K.S. Kuo, B.A. Wielicki, S.K. Sengupta, and L. Parker, Marine stratocumulus cloud fields off the coast of southem California observed by Landsat imagery, 1, Structural characteristics, J. Appl. Meteor., 27, 341-362, 1988a.

Welch, R.M., S.K. Sengupta, and K.S. Kuo, Marine stratocumulus cloud fields off the coast of southern California observed by Landsat imagery, 1, Textural analysis, J. Appl. Meteor., 27, 363-378, 1988 b.

Wendling, P., Albedo and reflected radiance of horizontally inhomogeneous clouds, J. Almos. Sci., 34, 642-650, 1977.

Wilson, J., D. Schertzer, and S. Lovejoy, Physically based rain and cloud modeling, in Scaling, Fractals and Non-Linear Variability in Geophysics, edited by D. Schertzer and S. Lovejoy, Kluwer, Hingham, Mass., in press, 1990.

Wiscombe, W.J., R.M. Welch, and W.D. Hall, The effects of very large drops on cloud absorption, 1, Parcel models, J. Atmos. Sci., 4l, 13361355. 1984.

Yano, J.-I., and Y. Takeuchi, Fractal dimension analysis of horizontal cloud pattern in the intertropical convergence zone, in Scaling, Fractals and Non-Linear Variability in Geophysics, edited by D. Schertzer and S. Lovejoy, Kluwer, Hingham, Mass., in press, 1990.

G. L. Austin and S. Lovejoy, Physics Department, McGill University, 3600 University Street, Montréal, Québec H3A-2T8, Canada. A. Davis and D. Schertzer, Météorologie Nationale, EERM/

CRMD, 2 Ave. Rapp, 75007 Paris, France.

P. Gabriel, CIRA, Dept. of Atmospheric Science, Colorado State University, Fort Collins, CO 80523.

(Received August 16, 1988;

revised May 17, 1989;

accepted September 19 1989.) 